\title{
A CLINICAL COMPARISON OF MODIFIED INSULINS 1,2
}

\author{
By JOSEPH L. IZZO 8 AND S. LEE CRUMP 4 WITH THE TECHNICAL ASSISTANCE OF \\ WERNER KUNZ \\ (From the Department of Medicine, University of Rochester, School of Medicine and Dentistry, \\ and the Medical Clinic of the Strong Memorial and Rochester Municipal Hospitals, \\ Rochester, New York)
}

(Submitted for publication April 25, 1950; accepted, August 21, 1950)

\section{INTRODUCTION}

The shortcomings of standard protamine zinc insulin when used alone in a single daily injection for the treatment of so-called "severe" diabetes are well known (1-4). Post-prandial glycosuria, nocturnal hypoglycemia, or both, are common and are directly attributed to its slow, gradual but variable rate of absorption from the subcutaneous depot (5). Consequently, increasing attention has been directed during the past several years toward developing a depot insulin whose activity would be distributed more in keeping with the physiological needs of the diabetic. Several preparations intermediate in time-action between ordinary unmodified insulin and standard protamine zinc insulin have been proposed. Of these the most promising and extensively studied are modifications of standard protamine zinc insulin (6-18) and globin insulin with zinc (19-32).

The modifications of standard protamine zinc insulin include premixed and extemporaneous mixtures of unmodified or crystalline insulin with standard protamine zinc insulin in various proportions (5-12), specially modified protamine zinc insulin, type NP-50 (13-15) and more recently specially modified insulin types NPC-40 and NPH50 (12). The most popular and widely used mixture seems to be the pre-mixed, unbuffered "2:1 mixture" (9-12), made by mixing thoroughly two parts of unmodified or crystalline insulin with one part of standard protamine zinc insulin. The physical nature and principal consti-

1 Aided by grants from Eli Lilly and Company, and from the Hochstetter Fund of the University of Rochester School of Medicine and Dentistry.

2 The insulins used in these studies were supplied through the courtesy of Eli Lilly and Company.

3 Bertha Hochstetter Fellow in Medicine, and Eli Lilly Fellow in Medicine.

4 From the Department of Mathematics, University of Rochester. tuents of the insulins mentioned are outlined in Table I.

In a previous report (33) the time action curves and solubility properties of all of these intermediate insulins were compared. This paper deals with a quantitative comparison of five of these insulins ${ }^{5}$ at the clinical level. No report has appeared previously in the literature in which all of these insulins have been compared clinically in the same group of patients.

\section{PLAN OF INVESTIGATION}

Patients. Nineteen women patients with diabetes mellitus were used in this investigation. Throughout the period of study, which in each case extended over several weeks, they lived quietly in the hospital under carefully controlled uniform conditions. They observed normal sleep and waking habits. Activity was restricted to being up and about on the hospital floors ad libitum.

The subjects selected do not represent a random sample of the population of our diabetic clinic. They were selected specifically to obtain a wide range in age, duration of diabetes, insulin requirements, and stability, as outlined in Table II. Since the classification of the patients on the basis of stability is important in the later interpretation of results it is pertinent to digress a moment to discuss it more fully.

It became obvious early in the investigation that despite meticulous control of the external factors the response in some cases was strikingly different from that in others. Observations of the types of patients under investigation impressed one of us with the fact that it is possible to separate out two rough clinical groups irrespective of amount of exogenous insulin required or type of insulin used. These have been called the relatively stable group (Patients 1-7) and the relatively unstable group (Patients 8-19). No sharp line of demarcation is possible between the groups. Nevertheless, in most instances a classification could be made without difficulty after a sufficient period of observation under controlled conditions.

In the unstable group control was difficult. Control is

5 NP-50 insulin was omitted because its manufacture had been discontinued at the time this investigation was started. 
TABLE I

Physical nature and principal constituents of different insulin preparations

\begin{tabular}{|c|c|c|c|c|c|c|c|}
\hline \multirow{2}{*}{ Name of preparation } & \multirow{2}{*}{$\mathrm{pH}$} & \multirow{2}{*}{$\begin{array}{l}\text { Physical } \\
\text { appear- } \\
\text { ance }\end{array}$} & \multirow{2}{*}{$\begin{array}{l}\text { Character } \\
\text { of ppt. }\end{array}$} & \multicolumn{2}{|c|}{ Modifying agent } & \multirow{2}{*}{ Zinc } & \multirow{2}{*}{$\begin{array}{l}\text { Relative amount of insulin in } \\
\text { solution and precipitate }\end{array}$} \\
\hline & & & & Type & Amt. & & \\
\hline & & & & & $\left(\begin{array}{c}\text { (mg./100u. } \\
\text { of insulin) }\end{array}\right.$ & & \\
\hline $\begin{array}{l}\text { Crystalline insulin } \\
\text { Standard protamine } \\
\text { zinc insulin }\end{array}$ & $\begin{array}{l}3.3 \\
7.2\end{array}$ & $\begin{array}{l}\text { Clear } \\
\text { Cloudy }\end{array}$ & $\begin{array}{l}\text { No ppt. } \\
\text { Amorphous }\end{array}$ & $\begin{array}{l}\text { None } \\
\text { Protamine }\end{array}$ & $\overline{1.25}$ & $\begin{array}{l}0.02 \\
0.20\end{array}$ & $\begin{array}{l}\text { All in solution } \\
\text { Practically all in the precipi- } \\
\text { tate }\end{array}$ \\
\hline $\begin{array}{l}2: 1 \text { mixture ( } 2 \text { parts } \\
\text { insulin, } 1 \text { part prota- } \\
\text { mine zinc insulin) }\end{array}$ & 5.9 & Cloudy & Amorphous & Protamine & 0.42 & 0.07 & $\begin{array}{l}\text { Practically all in the precipi- } \\
\text { tate }\end{array}$ \\
\hline $\begin{array}{l}\text { Special protamine zinc } \\
\text { insulin, type NP-50 }\end{array}$ & 7.2 & Cloudy & Amorphous & Protamine & 0.50 & & $\begin{array}{l}\text { Approximately } 25 \% \text { in solu- } \\
\text { tion and } 75 \% \text { in the pre- }\end{array}$ \\
\hline $\begin{array}{l}\text { Specially modified insu- } \\
\text { lin, type NPC-40 }\end{array}$ & 7.2 & Cloudy & Crystalline & Protamine & 0.40 & 0.02 & $\begin{array}{l}\text { Largely but not all in the } \\
\text { precipitate, a small fraction }\end{array}$ \\
\hline $\begin{array}{l}\text { Specially modified insu- } \\
\text { lin, type NPH-50 }\end{array}$ & 7.2 & Cloudy & Crystalline & Protamine & 0.50 & $\begin{array}{l}0.02- \\
0.05\end{array}$ & Practically all in the precipi- \\
\hline Globin insulin with zinc & 3.7 & Clear & No ppt. & Globin & 3.80 & 0.30 & All in solution \\
\hline
\end{tabular}

here used in the strict sense, implying not only complete absence of glycosuria but also maintenance of blood sugar levels within the normal range. Under the conditions of the study the typical unstable patient was subject to wide, unpredictable swings in blood sugar levels; complete absence of glycosuria was virtually impossible; intermittent or irregular glycosuria of variable degree was the rule; reactions to insulin tended to be frequent, severe, difficult to eliminate and aggravated by attempts to maintain normal blood sugar levels; ketosis was prone to develop rapidly; slight excesses or deficiencies of insulin were apt to produce marked hypo-or hyperglycemia, re- spectively. In contrast it was possible to approach control in the stable patient; variations in blood sugar levels were decidedly less; virtually complete absence of glycosuria was possible without the penalty of frequent or severe insulin reactions; the response of the blood sugar to changes in dose was sluggish; ketosis was not observed.

Diets. All patients received three weighed meals daily, breakfast at 8:00 a.m., lunch at 12:00 noon and supper at 5:00 p.m. In the relatively unstable group of patients in-between feedings at either 3:00 p.m., 9:30 p.m., or both were frequently necessary. For example, the evening feeding was routine with standard protamine zinc

TABLE II

Supplementary information about the patients in this study

\begin{tabular}{|c|c|c|c|c|c|c|c|c|}
\hline \multirow{2}{*}{$\begin{array}{l}\text { Patient } \\
\text { number }\end{array}$} & \multirow{2}{*}{ Age } & \multirow{2}{*}{$\begin{array}{l}\text { Duration of } \\
\text { diabetes }\end{array}$} & \multicolumn{4}{|c|}{ Diet } & \multicolumn{2}{|c|}{$\begin{array}{l}\text { Insulin-single daily dose } \\
\text { A.C. breakfast (units) }\end{array}$} \\
\hline & & & Protein & Fat & Carbohydrate & $\begin{array}{c}\text { Total } \\
\text { calories }\end{array}$ & $\begin{array}{l}\text { At start of } \\
\text { study }\end{array}$ & $\begin{array}{l}\text { At end of } \\
\text { study }\end{array}$ \\
\hline $\begin{array}{r}1 \\
2 \\
3 \\
4 \\
5 \\
6 \\
7 \\
8 \\
9 \\
10 \\
11 \\
12 \\
13 \\
14 \\
15 \\
16 \\
17 \\
18 \\
19\end{array}$ & $\begin{array}{c}(y r s) \\
49 \\
74 \\
66 \\
60 \\
74 \\
74 \\
67 \\
44 \\
53 \\
21 \\
22 \\
39 \\
58 \\
66 \\
14 \\
21 \\
28 \\
55 \\
24\end{array}$ & $\begin{array}{c}(y r s) \\
14 \\
20 \\
1 \frac{1}{3} \\
10 \\
7 \\
4 \\
{ }^{\frac{1}{2}} \\
14^{\frac{3}{3}} \\
7 \\
1 \\
1 \frac{1}{2} \\
10 \\
5 \\
13 \\
3 \\
6 \\
23 \\
24 \\
5\end{array}$ & $\begin{array}{c}\text { (in g.) } \\
75 \\
69 \\
63 \\
78 \\
70 \\
70 \\
87 \\
81 \\
72 \\
92 \\
81 \\
69 \\
94 \\
77 \\
90 \\
95 \\
60 \\
71 \\
76\end{array}$ & $\begin{array}{r}\text { (in g.) } \\
40 \\
72 \\
60 \\
78 \\
96 \\
90 \\
98 \\
84 \\
46 \\
94 \\
94 \\
138 \\
106 \\
93 \\
100 \\
101 \\
71 \\
100 \\
72\end{array}$ & $\begin{array}{c}\text { (in g.) } \\
151 \\
138 \\
102 \\
150 \\
170 \\
170 \\
183 \\
160 \\
150 \\
171 \\
181 \\
164 \\
195 \\
188 \\
248 \\
200 \\
182 \\
150 \\
171\end{array}$ & $\begin{array}{r}1,264 \\
1,476 \\
900 \\
1,534 \\
1,824 \\
1,770 \\
1,962 \\
1,720 \\
1,392 \\
1,898 \\
1,898 \\
2,674 \\
2,010 \\
1,897 \\
2,260 \\
2,170 \\
1,607 \\
1,784 \\
1,546\end{array}$ & $\begin{array}{r}70 \\
65 \\
75 \\
44 \\
44 \\
35 \\
15 \\
20 \\
50 \\
240 \\
48 \\
24 \\
48 \\
56 \\
75 \\
75 \\
48 \\
28 \\
40\end{array}$ & $\begin{array}{r}45 \\
55 \\
60 \\
44 \\
44 \\
35 \\
35 \\
22 \\
30 \\
200 \\
46 \\
30 \\
40 \\
56 \\
140 \\
50 \\
52 \\
34 \\
55\end{array}$ \\
\hline
\end{tabular}


insulin while the mid-afternoon feeding was routine with globin insulin with zinc. The pattern of in-between feedings was less consistent with the modifications of standard protamine zinc insulin. As a rule the diets (see Table II) approximated those that the patients were accustomed to receiving prior to the investigation. The protein value of the diet was calculated on the basis of 1.0 to $1.5 \mathrm{~g}$. per $\mathrm{kg}$. of ideal body weight and the remaining calories distributed approximately equally between carbohydrate and fat. In the sub-maintenance diets reduction in calories was chiefly at the expense of fat. The total daily carbohydrate intake in most cases ranged between $150 \mathrm{~g}$. and $200 \mathrm{~g}$., with extremes of $102 \mathrm{~g}$. in one elderly patient on a very low caloric intake and $248 \mathrm{~g}$. in an adolescent patient. The basic distribution of carbohydrate for the three meals was breakfast $20 \%$, lunch $40 \%$, and supper $40 \%$, with lunch and supper both contributing to the in-between feedings. The range of carbohydrate distribution was as follows : breakfast $20-30 \%$, lunch $35-40 \%$, supper $30-40 \%$, in-between feeding $8-15 \%$.

Insulins. Each of the patients was observed from one to six weeks in the hospital under uniform conditions before the insulin comparisons were begun. It was found that for a given patient the over-all variability among blood and urinary sugar levels remained quite constant during this period. Since the insulins were to be compared on their ability to affect this variability it was felt that a more extended initial period of adjustment would not have altered the results of the insulin comparisons.

Five different insulins, namely: the $2: 1$ mixture, NPC-40 insulin, NPH-50 insulin, globin insulin with zinc, and standard protamine zinc insulin were compared in each patient. The order in which they were given was varied from patient to patient. Insulin was always administered hypodermically in a single daily dose before breakfast. The comparisons are based on the performance of each insulin over periods of three to ten consecutive days (mode of five days). On changing from one insulin to another a period of adjustment (usually one to three days) was allowed before the comparison was made. In most patients the performances of one or more of the insulins were observed during two periods separated by at least one period on some other insulin. With few exceptions it was found impossible to maintain the insulin dose in the individual patient constant throughout the study. Changes in tolerance necessitated slight adjustments in dose from time to time. In some cases a gradual improvement in tolerance occurred so that the insulin dose had to be gradually reduced; in others, however, the reverse was true. In six patients (nos. 10,14, 15, 17, 18, 19) standard protamine zinc insulin was omitted because of a history of severe nocturnal reactions with doses large enough to prevent marked glycosuria or ketosis during the day. It was not felt advisable to subject these individuals to the possibility of severe nocturnal reactions.

Blood and urinary sugar. Specimens of venous blood were taken four times daily at 7:30 a.m., 11:30 a.m., 4:30 p.m., and 9:30 p.m., and analyzed for glucose according to Somogyi's modification of the Shaffer-Hartman copper iodometric titration method for "true" blood sugar estimation (34). Twenty-four hour urines were collected in four periods: $7: 30-11: 30$ a.m., $11: 30-4: 30$ p.m., $4: 30-9: 30$ p.m., and $9: 30$ p.m. $-7: 30$ a.m., and analyzed quantitatively for glucose according to the Shaffer-Hartman method (35) using Somogyi's new copper reagent (34).

\section{ANALYSIS OF THE DATA}

The volume of the original data makes it impractical to present them in their entirety. A summary of the original data is presented in Tables III and IV. The purpose of these tables is only to provide the reader with a general impression of the levels and variability of the blood and urinary sugar determinations during the period of study.

The primary problem in the analysis of these data is a descriptive one. The extent of the data makes it difficult to determine by inspection whether there are consistencies of patient response reflected by blood and urinary sugar levels which relate to type of insulin received. A striking feature in the blood and urinary sugar levels in diabetics is their variability in contrast to their constancy in non-diabetics. Hence, a reasonable basis for comparing insulins is their effect on this variability. The total variability among the blood or urinary sugar levels for each patient on each insulin has been divided into two components, intraand inter-daily variability. The intra-daily variation is the variability among the four determinations (of blood or urinary sugar) in a single day. To measure this variation monotonic functions of the variance ${ }^{6}$ of the four readings in a single day have been used. Precise definitions of intra-daily variation for blood and urinary sugar are given in the appendix.

The inter-daily variation is the variability among the average blood or urinary sugar levels from one day to another. In order to compare the different kinds of insulin it would not be pertinent.to have the measure of inter-daily variation include variability resulting from changes in unit dose from one day to the next. Inter-daily variation should include only that variability remaining when the patient is treated as nearly identically as pos-

6 The variance of a set of numbers is an average of the squared differences between the numbers and their mean. The use of the variance as a measure of variability is discussed in any standard text on statistics, e.g., Snedecor (36). 
TABLE III

Summary of blood sugar data

\begin{tabular}{|c|c|c|c|c|c|c|c|}
\hline \multirow{2}{*}{ Patient } & \multirow{2}{*}{ Insulin } & \multirow{2}{*}{$\begin{array}{l}\text { Average } \\
\text { daily } \\
\text { dose }\end{array}$} & \multicolumn{4}{|c|}{ Mean and range of blood sugar in $\mathrm{mg} . \%$ at } & \multirow{2}{*}{$\begin{array}{l}\text { Number } \\
\text { of days } \\
\text { of obser- } \\
\text { vation }\end{array}$} \\
\hline & & & 7:30 a.m. & $11: 30$ a.m. & 4:30 p.m. & 9:30 p.m. & \\
\hline 1 & $\begin{array}{l}2: 1 \text { mixture } \\
\text { NPC-40 } \\
\text { NPH-50 } \\
\text { Protamine zinc } \\
\text { Globin with zinc }\end{array}$ & $\begin{array}{l}63 \\
45 \\
52 \\
70 \\
47\end{array}$ & $\begin{array}{l}106(99-115) \\
136(120-150) \\
129(108-140) \\
138(134-155) \\
131(115-146)\end{array}$ & $\begin{array}{c}90(79-107) \\
121(103-144) \\
104(73-131) \\
127(117-141) \\
121(97-138)\end{array}$ & $\begin{array}{r}85(70-112) \\
116(97-129) \\
96(74-127) \\
119(77-148) \\
100(56-132)\end{array}$ & $\begin{array}{l}104(98-110) \\
134(124-141) \\
114(82-134) \\
147(100-190) \\
107(82-127)\end{array}$ & $\begin{array}{l}3 \\
3 \\
6 \\
5 \\
3\end{array}$ \\
\hline 2 & $\begin{array}{l}2: 1 \text { mixture } \\
\text { NPC-40 } \\
\text { NPH-50 } \\
\text { Protamine zinc } \\
\text { Globin with zinc }\end{array}$ & $\begin{array}{l}61 \\
59 \\
56 \\
60 \\
60\end{array}$ & $\begin{array}{r}99(76-133) \\
116(84-159) \\
121(47-163) \\
92(75-104) \\
122(99-165)\end{array}$ & $\begin{array}{r}92(52-189) \\
94(60-140) \\
109(88-127) \\
108(86-131) \\
126(99-157)\end{array}$ & $\begin{array}{l}93(68-148) \\
107(78-148) \\
127(86-197) \\
130(113-151) \\
136(104-162)\end{array}$ & $\begin{array}{l}132(105-246) \\
144(109-200) \\
172(145-197) \\
158(137-179) \\
154(130-171)\end{array}$ & $\begin{array}{r}12 \\
10 \\
14 \\
6 \\
6\end{array}$ \\
\hline 3 & $\begin{array}{l}2: 1 \text { mixture } \\
\text { NPC-40 } \\
\text { NPH-50 } \\
\text { Protamine zinc } \\
\text { Globin with zinc }\end{array}$ & $\begin{array}{l}65 \\
60 \\
60 \\
72 \\
60\end{array}$ & $\begin{array}{l}135(88-176) \\
147(130-166) \\
151(123-171) \\
114(65-164) \\
138(88-172)\end{array}$ & $\begin{array}{l}141(104-161) \\
155(144-178) \\
164(148-182) \\
128(82-171) \\
133(128-138)\end{array}$ & $\begin{array}{l}118(93-144) \\
115(75-164) \\
138(116-170) \\
124(86-162) \\
140(130-148)\end{array}$ & $\begin{array}{l}126(43-162) \\
128(98-150) \\
151(136-173) \\
140(116-176) \\
104(68-154)\end{array}$ & $\begin{array}{l}4 \\
4 \\
4 \\
4 \\
4\end{array}$ \\
\hline 4 & $\begin{array}{l}2: 1 \text { mixture } \\
\text { NPC }-40 \\
\text { NPH-50 } \\
\text { Protamine zinc } \\
\text { Globin with zinc }\end{array}$ & $\begin{array}{l}44 \\
44 \\
45 \\
44 \\
44\end{array}$ & $\begin{array}{l}162(143-193) \\
152(139-164) \\
160(100-188) \\
165(121-200) \\
142(106-159)\end{array}$ & $\begin{array}{l}180(167-204) \\
161(142-176) \\
184(89-270) \\
161(78-224) \\
218(199-237)\end{array}$ & $\begin{array}{l}172(151-208) \\
175(146-200) \\
198(168-238) \\
212(138-251) \\
210(186-226)\end{array}$ & $\begin{array}{l}201(172-246) \\
185(144-224) \\
234(194-264) \\
193(85-258) \\
157(128-185)\end{array}$ & $\begin{array}{l}3 \\
3 \\
6 \\
3 \\
3\end{array}$ \\
\hline 5 & $\begin{array}{l}2: 1 \text { mixture } \\
\text { NPC }-40 \\
\text { NPH-50 } \\
\text { Protamine zinc } \\
\text { Globin with zinc }\end{array}$ & $\begin{array}{l}44 \\
44 \\
44 \\
44 \\
44\end{array}$ & $\begin{array}{l}152(119-195) \\
186(139-217) \\
165(133-229) \\
146(97-193) \\
134(118-146)\end{array}$ & $\begin{array}{l}166(148-184) \\
178(54-233) \\
194(154-248) \\
165(127-200) \\
171(161-188)\end{array}$ & $\begin{array}{l}146(99-168) \\
169(107-217) \\
178(121-265) \\
170(124-208) \\
147(111-184)\end{array}$ & $\begin{array}{l}189(162-211) \\
235(177-268) \\
235(165-350) \\
206(182-224) \\
171(129-198)\end{array}$ & $\begin{array}{l}5 \\
6 \\
8 \\
5 \\
5\end{array}$ \\
\hline 6 & $\begin{array}{l}2: 1 \text { mixture } \\
\text { NPC }-40 \\
\text { NPH-50 } \\
\text { Protamine zinc } \\
\text { Globin with zinc }\end{array}$ & $\begin{array}{l}35 \\
35 \\
35 \\
35 \\
35\end{array}$ & $\begin{array}{l}112(103-121) \\
126(99-165) \\
128(112-137) \\
106(97-112) \\
100(78-117)\end{array}$ & $\begin{array}{c}120(103-136) \\
148(137-157) \\
158(133-182) \\
97(84-112) \\
120(97-144)\end{array}$ & $\begin{array}{l}140(88-193) \\
114(88-130) \\
138(124-166) \\
118(70-142) \\
141(131-150)\end{array}$ & $\begin{array}{l}126(75-152) \\
146(107-194) \\
172(163-190) \\
127(95-144) \\
152(126-179)\end{array}$ & $\begin{array}{l}3 \\
3 \\
3 \\
3 \\
3\end{array}$ \\
\hline 7 & $\begin{array}{l}2: 1 \text { mixture } \\
\text { NPC }-40 \\
\text { NPH-50 } \\
\text { Protamine zinc } \\
\text { Globin with zinc }\end{array}$ & $\begin{array}{l}26 \\
24 \\
23 \\
23 \\
24\end{array}$ & $\begin{array}{l}136(97-193) \\
159(82-200) \\
154(132-182) \\
148(94-222) \\
168(113-216)\end{array}$ & $\begin{array}{l}148(60-218) \\
172(99-266) \\
174(56-252) \\
212(78-318) \\
172(104-230)\end{array}$ & $\begin{array}{c}146(53-252) \\
99(66-127) \\
137(90-199) \\
230(154-286) \\
154(76-210)\end{array}$ & $\begin{array}{l}171(73-260) \\
149(110-170) \\
160(96-213) \\
226(139-295) \\
160(62-248)\end{array}$ & $\begin{array}{l}8 \\
4 \\
7 \\
7 \\
7\end{array}$ \\
\hline 8 & $\begin{array}{l}2: 1 \text { mixture } \\
\text { NPC }-40 \\
\text { NPH-50 } \\
\text { Protamine zinc } \\
\text { Globin with zinc }\end{array}$ & $\begin{array}{l}18 \\
18 \\
20 \\
17 \\
20\end{array}$ & $\begin{array}{l}184(146-234) \\
135(108-160) \\
176(86-254) \\
163(112-197) \\
206(150-259)\end{array}$ & $\begin{array}{l}183(129-233) \\
154(92-254) \\
185(51-290) \\
200(162-282) \\
208(120-295)\end{array}$ & $\begin{array}{l}203(152-243) \\
114(74-144) \\
172(14-270) \\
181(140-204) \\
180(84-226)\end{array}$ & $\begin{array}{l}227(132-296) \\
160(105-198) \\
211(160-314) \\
268(135-454) \\
236(185-299)\end{array}$ & $\begin{array}{r}4 \\
4 \\
10 \\
4 \\
8\end{array}$ \\
\hline 9 & $\begin{array}{l}2: 1 \text { mixture } \\
\text { NPC-40 } \\
\text { NPH-50 } \\
\text { Protamine zinc } \\
\text { Globin with zinc }\end{array}$ & $\begin{array}{l}45 \\
40 \\
39 \\
36 \\
44\end{array}$ & $\begin{array}{c}124(80-179) \\
132(85-175) \\
107(47-222) \\
68(59-77) \\
166(93-264)\end{array}$ & $\begin{array}{l}157(71-260) \\
185(157-233) \\
146(65-203) \\
147(110-198) \\
198(136-251)\end{array}$ & $\begin{array}{l}109(40-154) \\
103(68-132) \\
113(63-243) \\
168(140-204) \\
116(47-219)\end{array}$ & $\begin{array}{r}129(53-255) \\
88(46-129) \\
91(28-220) \\
127(93-159) \\
72(49-130)\end{array}$ & $\begin{array}{r}7 \\
4 \\
10 \\
3 \\
7\end{array}$ \\
\hline \multirow[t]{2}{*}{10} & $\begin{array}{l}2: 1 \text { mixture } \\
\text { NPC-40 } \\
\text { NPH-50 } \\
\text { Protamine zinc }\end{array}$ & $\begin{array}{l}240 \\
216 \\
220\end{array}$ & $\begin{array}{l}91(68-128) \\
69(27-86) \\
80(57-116)\end{array}$ & $\begin{array}{l}167(115-239) \\
124(76-184) \\
106(60-178)\end{array}$ & $\begin{array}{c}136(57-276) \\
54(26-84) \\
91(60-201)\end{array}$ & $\begin{array}{r}130(74-212) \\
72(43-128) \\
100(23-205)\end{array}$ & $\begin{array}{l}4 \\
5 \\
8\end{array}$ \\
\hline & Globin with zinc & 200 & $84(51-128)$ & $167(106-269)$ & $100(24-187)$ & $52(31-79)$ & 4 \\
\hline 11 & $\begin{array}{l}\text { 2:1 mixture } \\
\text { NPC-40 } \\
\text { NPH-50 } \\
\text { Protamine zinc } \\
\text { Globin with zinc }\end{array}$ & $\begin{array}{l}49 \\
50 \\
49 \\
42 \\
48\end{array}$ & $\begin{array}{r}120(67-160) \\
107(83-144) \\
135(87-182) \\
87(21-139) \\
131(95-174)\end{array}$ & $\begin{array}{l}151(41-229) \\
112(53-152) \\
115(56-162) \\
178(116-238) \\
103(40-148)\end{array}$ & $\begin{array}{c}102(29-194) \\
110(47-167) \\
93(44-148) \\
157(108-195) \\
78(62-85)\end{array}$ & $\begin{array}{l}120(54-219) \\
119(64-170) \\
108(55-163) \\
146(70-206) \\
132(68-217)\end{array}$ & $\begin{array}{r}14 \\
6 \\
6 \\
5 \\
6\end{array}$ \\
\hline
\end{tabular}


TABLE III-Continued

\begin{tabular}{|c|c|c|c|c|c|c|c|}
\hline \multirow{2}{*}{ Patient } & \multirow{2}{*}{ Insulin } & \multirow{2}{*}{$\begin{array}{c}\text { Average } \\
\text { daily } \\
\text { dose }\end{array}$} & \multicolumn{4}{|c|}{ Mean and range of blood sugar in mg. \% at } & \multirow{2}{*}{$\begin{array}{l}\text { Number } \\
\text { of days } \\
\text { of obser- } \\
\text { vation }\end{array}$} \\
\hline & & & 7:30 a.m. & $11: 30$ a.m. & 4:30 p.m. & 9:30 p.m. & \\
\hline 12 & $\begin{array}{l}2: 1 \text { mixture } \\
\text { NPC-40 } \\
\text { NPH-50 } \\
\text { Protamine zinc } \\
\text { Globin with zinc }\end{array}$ & $\begin{array}{l}30 \\
30 \\
30 \\
28 \\
30\end{array}$ & $\begin{array}{r}83(70-92) \\
118(88-169) \\
106(79-167) \\
93(73-115) \\
111(91-150)\end{array}$ & $\begin{array}{c}94(72-146) \\
106(50-132) \\
107(53-229) \\
198(159-225) \\
173(146-232)\end{array}$ & $\begin{array}{l}116(56-175) \\
131(67-167) \\
140(69-256) \\
294(166-374) \\
102(55-157)\end{array}$ & $\begin{array}{l}111(99-119) \\
146(98-212) \\
137(47-232) \\
188(120-288) \\
101(61-131)\end{array}$ & $\begin{array}{l}3 \\
3 \\
7 \\
4 \\
4\end{array}$ \\
\hline 13 & $\begin{array}{l}2: 1 \text { mixture } \\
\text { NPC-40 } \\
\text { NPH-50 } \\
\text { Protamine zinc } \\
\text { Globin with zinc }\end{array}$ & $\begin{array}{l}65 \\
49 \\
55 \\
47 \\
40\end{array}$ & $\begin{array}{l}200(176-242) \\
209(177-240) \\
169(77-208) \\
176(77-268) \\
175(88-248)\end{array}$ & $\begin{array}{l}142(95-214) \\
137(68-203) \\
178(88-257) \\
212(107-318) \\
174(43-257)\end{array}$ & $\begin{array}{l}198(136-263) \\
161(62-323) \\
157(69-252) \\
241(104-337) \\
100(48-205)\end{array}$ & $\begin{array}{l}242(204-291) \\
176(126-228) \\
176(116-353) \\
239(51-428) \\
155(102-198)\end{array}$ & $\begin{array}{r}4 \\
8 \\
13 \\
8 \\
8\end{array}$ \\
\hline 14 & $\begin{array}{l}2: 1 \text { mixture } \\
\text { NPC-40 } \\
\text { NPH-50 } \\
\text { Protamine zinc } \\
\text { Globin with zinc }\end{array}$ & $\begin{array}{l}54 \\
55 \\
55 \\
56\end{array}$ & $\begin{array}{l}148(56-289) \\
140(61-196) \\
175(31-329) \\
251(82-344)\end{array}$ & $\begin{array}{l}203(66-331) \\
183(82-263) \\
262(82-342) \\
170(61-356)\end{array}$ & $\begin{array}{r}148(52-279) \\
138(64-213) \\
207(99-352) \\
83(46-185)\end{array}$ & $\begin{array}{l}153(33-269) \\
163(78-222) \\
265(141-343) \\
205(139-293)\end{array}$ & $\begin{array}{r}14 \\
8 \\
9 \\
6\end{array}$ \\
\hline 15 & $\begin{array}{l}2: 1 \text { mixture } \\
\text { NPC-40 } \\
\text { NPH-50 } \\
\text { Protamine zinc } \\
\text { Globin with zinc }\end{array}$ & $\begin{array}{l}116 \\
140 \\
150 \\
140\end{array}$ & $\begin{array}{l}225(82-340) \\
100(55-147) \\
126(82-170) \\
151(90-225)\end{array}$ & $\begin{array}{c}237(51-312) \\
50(49-61) \\
114(53-238) \\
100(63-120)\end{array}$ & $\begin{array}{c}235(107-405) \\
195(57-279) \\
226(166-326) \\
83(37-123)\end{array}$ & $\begin{array}{l}332(240-415) \\
238(140-313) \\
190(153-262) \\
247(109-328)\end{array}$ & $\begin{array}{r}17 \\
4 \\
4 \\
3\end{array}$ \\
\hline 16 & $\begin{array}{l}2: 1 \text { mixture } \\
\text { NPC-40 } \\
\text { NPH-50 } \\
\text { Protamine zinc } \\
\text { Globin with zinc }\end{array}$ & $\begin{array}{l}75 \\
55 \\
60 \\
49 \\
55\end{array}$ & $\begin{array}{r}89(27-228) \\
129(34-288) \\
104(43-286) \\
114(40-239) \\
184(51-276)\end{array}$ & $\begin{array}{r}88(39-176) \\
72(34-132) \\
99(21-227) \\
120(34-236) \\
138(25-274)\end{array}$ & $\begin{array}{r}99(23-214) \\
111(26-214) \\
99(31-255) \\
178(73-318) \\
73(32-123)\end{array}$ & $\begin{array}{l}152(33-310) \\
131(29-276) \\
193(67-322) \\
225(73-356) \\
142(69-257)\end{array}$ & $\begin{array}{l}6 \\
5 \\
9 \\
4 \\
5\end{array}$ \\
\hline 17 & $\begin{array}{l}2: 1 \text { mixture } \\
\text { NPC-40 } \\
\text { NPH-50 } \\
\text { Protamine zinc } \\
\text { Globin with zinc }\end{array}$ & $\begin{array}{l}48 \\
50 \\
52 \\
48\end{array}$ & $\begin{array}{l}129(38-237) \\
189(40-410) \\
150(27-304) \\
381(328-475)\end{array}$ & $\begin{array}{l}137(37-306) \\
160(64-274) \\
200(58-343) \\
311(87-398)\end{array}$ & $\begin{array}{c}152(27-291) \\
172(35-384) \\
202(31-366) \\
76(8-206)\end{array}$ & $\begin{array}{r}110(28-190) \\
240(70-436) \\
213(22-376) \\
71(31-169)\end{array}$ & $\begin{array}{r}5 \\
10 \\
5 \\
5\end{array}$ \\
\hline 18 & $\begin{array}{l}2: 1 \text { mixture } \\
\text { NPC-40 } \\
\text { NPH-50 } \\
\text { Protamine zinc } \\
\text { Globin with zinc }\end{array}$ & $\begin{array}{l}30 \\
32 \\
33 \\
34\end{array}$ & $\begin{array}{l}246(20-359) \\
220(45-360) \\
183(109-347) \\
214(105-340)\end{array}$ & $\begin{array}{l}181(16-285) \\
199(34-348) \\
138(12-260) \\
153(26-339)\end{array}$ & $\begin{array}{l}119(28-296) \\
234(134-377) \\
133(25-278) \\
109(42-265)\end{array}$ & $\begin{array}{l}194(82-237) \\
243(145-437) \\
177(70-307) \\
150(48-365)\end{array}$ & $\begin{array}{l}9 \\
7 \\
6 \\
4\end{array}$ \\
\hline 19 & $\begin{array}{l}2: 1 \text { mixture } \\
\text { NPC }-40 \\
\text { NPH-50 } \\
\text { Protamine zinc } \\
\text { Globin with zinc }\end{array}$ & $\begin{array}{l}51 \\
56 \\
46\end{array}$ & $\begin{array}{l}162(70-269) \\
148(73-282) \\
110(64-221) \\
266(145-346)\end{array}$ & $\begin{array}{l}286(208-374) \\
229(160-285) \\
212(93-300)\end{array}$ & $\begin{array}{l}262(88-460) \\
270(196-357) \\
205(118-258)\end{array}$ & $\begin{array}{l}214(53-274) \\
241(119-347) \\
228(159-314) \\
\\
111(53-226)\end{array}$ & $\begin{array}{r}8 \\
4 \\
10 \\
\\
5\end{array}$ \\
\hline
\end{tabular}

sible for each of a series of days. The period of observation for each patient while receiving one kind of insulin has been divided into "groups" of days. A "group" of days is a series of consecutive days on which a single patient received the same unit dose of the same kind of insulin. Inter-daily variation is then measured separately for each "group" of days in a manner similar to that for intra-daily variation. Precise definitions of interdaily variation are given in the appendix.

The choice of particular measures of intra- and inter-daily variability is to a large extent arbitrary. The measures used in this analysis were chosen because they possess certain desirable statistical properties.

Each patient provides one estimate of her intradaily variation in both blood and urinary sugar on each day of observation. When these are averaged over all days on which the patient received the same kind of insulin, the insulins may be compared on their ability to control intra-daily variability. Similarly, each patient provides one estimate of her inter-daily variation during each "group" of days of observation. These may be averaged over all "groups" of days during which the patient received the same kind of insulin in order to com- 
pare insulins on their ability to control inter-daily variability.

While intra-daily variation measures the extent of the variability among the four blood sugar determinations in a single day it fails to describe the pattern of this variability. It is possible for two insulins to produce no detectable difference in intradaily variation even though they produce clearly different patterns. In order to describe the pattern of the intra-daily variability the mean deviation from the daily mean at the four specified times of the day has been calculated for each patient on each insulin. For example, at 7:30 a.m. the mean deviation from the daily mean ${ }^{7}$ is the aver-

7 The mean deviations have also been calculated for the urinary sugar determinations. Since they reflect the age of the deviations of blood sugar values at that time from their corresponding daily means, where the averaging is over all days on which the patient received a specified insulin.

Statistical tests of significance have been utilized in deciding whether observed differences in averages are sufficiently large to warrant the conclusion that kind of insulin had a real influence on the quantity in question. In all cases the 5\% significance level has been used and the tests have been carried out by means of Analysis of Variance Technique. For a full discussion of this statistical method see Snedecor (36). It has been assumed throughout that changes in dose within the ranges

same patterns as the blood sugars they have not been presented.

TABLE IV

Summary of urinary sugar data

\begin{tabular}{|c|c|c|c|c|c|c|c|c|}
\hline \multirow{2}{*}{ Patient } & \multirow{2}{*}{ Insulin } & \multirow{2}{*}{$\begin{array}{l}\text { Aver- } \\
\text { age } \\
\text { daily } \\
\text { dose }\end{array}$} & \multicolumn{4}{|c|}{ Mean and range of urinary sugar in $\mathrm{g}$. from } & \multirow{2}{*}{$\begin{array}{l}\text { Daily } \\
\text { total }\end{array}$} & \multirow{2}{*}{$\begin{array}{l}\text { No. of } \\
\text { days of } \\
\text { obser- } \\
\text { vation }\end{array}$} \\
\hline & & & $\begin{array}{l}7.30 \text { a.m.- } \\
11: 30 \text { a.m. }\end{array}$ & $\begin{array}{c}11: 30 \text { a.m.- } \\
\text { 4:30 p.m. }\end{array}$ & $\begin{array}{l}\text { 4:30 p.m.- } \\
\text { 9:30 p.m. }\end{array}$ & $\begin{array}{l}\text { 9:30 p.m.- } \\
\text { 7:30 a.m. }\end{array}$ & & \\
\hline 1 & $\begin{array}{l}2: 1 \text { mixture } \\
\text { NPC }-40 \\
\text { NPH-50 } \\
\text { Protamine zinc } \\
\text { Globin with zinc }\end{array}$ & $\begin{array}{l}63 \\
45 \\
52 \\
70 \\
47\end{array}$ & $\begin{array}{l}0.15(0.13-0.17) \\
0.16(0.12-0.21) \\
0.54(0.10-2.38) \\
0.22(0.15-0.40) \\
0.15(0.03-0.24)\end{array}$ & $\begin{array}{l}0.15(0.13-0.17) \\
0.15(0.11-0.21) \\
0.25(0.09-0.65) \\
0.20(0.15-0.30) \\
0.10(0.00-0.24)\end{array}$ & $\begin{array}{l}0.15(0.13-0.17) \\
0.15(0.09-0.21) \\
0.44(0.10-1.78) \\
0.20(0.15-0.30) \\
0.14(0.03-0.20)\end{array}$ & $\begin{array}{l}0.17(0.15-0.20) \\
0.16(0.11-0.22) \\
0.68(0.09-2.83) \\
0.38(0.15-1.02) \\
0.21(0.08-0.28)\end{array}$ & $\begin{array}{l}0.62(0.54-0.71) \\
0.62(0.43-0.85) \\
1.91(0.39-7.64) \\
0.99(0.60-2.02) \\
0.61(0.12-0.96)\end{array}$ & $\begin{array}{l}3 \\
3 \\
6 \\
5 \\
3\end{array}$ \\
\hline 2 & $\begin{array}{l}2: 1 \text { mixture } \\
\text { NPC }-40 \\
\text { NPH-50 } \\
\text { Protamine zinc } \\
\text { Globin with zinc }\end{array}$ & $\begin{array}{l}61 \\
58 \\
56 \\
60 \\
60\end{array}$ & $\begin{array}{l}0.16(0.02-0.82) \\
0.34(0.08-1.90) \\
0.26(0.02-0.91) \\
0.16(0.05-0.31) \\
0.23(0.09-0.45)\end{array}$ & $\begin{array}{l}0.32(0.00-1.50) \\
0.22(0.09-i .00) \\
0.18(0.02-0.35) \\
0.20(0.10-0.36) \\
0.35(0.14-0.73)\end{array}$ & $\begin{array}{l}0.28(0.12-1.59) \\
0.26(0.06-0.50) \\
0.24(0.05-0.43) \\
0.26(0.14-0.45) \\
0.18(0.12-0.29)\end{array}$ & $\begin{array}{l}0.30(0.13-0.58) \\
0.26(0.14-0.40) \\
0.37(0.06-0.58) \\
0.26(0.07-0.38) \\
0.34(0.27-0.45)\end{array}$ & $\begin{array}{l}1.08(0.26-4.49) \\
1.04(0.39-3.70) \\
1.04(0.19-1.89) \\
0.88(0.69-1.28) \\
1.11(0.75-1.69)\end{array}$ & $\begin{array}{r}12 \\
8 \\
13 \\
6 \\
6\end{array}$ \\
\hline 3 & $\begin{array}{l}2: 1 \text { mixture } \\
\text { NPC }-40 \\
\text { NPH-50 } \\
\text { Protamine zinc } \\
\text { Globin with zinc }\end{array}$ & $\begin{array}{l}65 \\
60 \\
60 \\
72 \\
60\end{array}$ & $\begin{array}{l}0.11(0.08-0.12) \\
0.12(0.10-0.19) \\
0.14(0.04-0.32) \\
0.12(0.06-0.20) \\
0.15(0.09-0.21)\end{array}$ & $\begin{array}{l}0.11(0.08-0.13) \\
0.13(0.10-0.21) \\
0.16(0.10-0.30) \\
0.12(0.05-0.20) \\
0.12(0.09-0.17)\end{array}$ & $\begin{array}{l}0.16(0.08-0.27) \\
0.10(0.09-0.11) \\
0.14(0.10-0.22) \\
0.13(0.07-0.18) \\
0.18(0.09-0.27)\end{array}$ & $\begin{array}{l}0.22(0.08-0.45) \\
0.16(0.08-0.33) \\
0.11(0.09-0.15) \\
0.49(0.13-1.16) \\
0.23(0.08-0.62)\end{array}$ & $\begin{array}{l}0.59(0.32-0.97) \\
0.51(0.38-0.82) \\
0.56(0.39-0.73) \\
0.86(0.47-1.39) \\
0.67(0.35-1.24)\end{array}$ & $\begin{array}{l}3 \\
4 \\
4 \\
4 \\
4\end{array}$ \\
\hline 4 & $\begin{array}{l}2: 1 \text { mixture } \\
\text { NPC }-40 \\
\text { NPH-50 } \\
\text { Protamine zinc } \\
\text { Globin with zinc }\end{array}$ & $\begin{array}{l}44 \\
44 \\
45 \\
44 \\
44\end{array}$ & $\begin{array}{l}0.07(0.06-0.08) \\
0.18(0.04-0.33) \\
1.18(0.04-2.72) \\
1.13(0.93-1.45) \\
0.36(0.08-0.82)\end{array}$ & $\begin{array}{l}0.18(0.06-0.30) \\
0.22(0.16-0.31) \\
0.88(0.08-1.72) \\
0.84(0.06-1.92) \\
0.71(0.08-1.85)\end{array}$ & $\begin{array}{l}0.14(0.06-0.22) \\
0.14(0.08-0.19) \\
0.81(0.08-1.93) \\
0.72(0.50-0.85) \\
0.38(0.08-0.89)\end{array}$ & $\begin{array}{l}0.25(0.07-0.43) \\
0.43(0.13-0.99) \\
0.85(0.08-3.10) \\
1.22(0.13-2.68) \\
1.18(0.08-2.16)\end{array}$ & $\begin{array}{l}0.64(0.25-1.03) \\
0.96(0.43-1.82) \\
3.71(0.32-7.31) \\
3.91(1.97-6.55) \\
2.63(0.32-4.86)\end{array}$ & $\begin{array}{l}2 \\
3 \\
6 \\
3 \\
3\end{array}$ \\
\hline 5 & $\begin{array}{l}2: 1 \text { mixture } \\
\text { NPC-40 } \\
\text { NPH-50 } \\
\text { Protamine zinc } \\
\text { Globin with zinc }\end{array}$ & $\begin{array}{l}44 \\
44 \\
44 \\
44 \\
44\end{array}$ & $\begin{array}{l}0.20(0.08-0.41) \\
0.49(0.16-0.70) \\
0.58(0.01-1.97) \\
0.31(0.10-0.71) \\
0.38(0.16-0.77)\end{array}$ & $\begin{array}{l}0.34(0.13-0.97) \\
2.63(0.15-6.39) \\
0.66(0.01-2.14) \\
0.21(0.03-0.35) \\
0.34(0.05-0.68)\end{array}$ & $\begin{array}{l}0.17(0.10-0.29) \\
0.25(0.13-0.52) \\
0.46(0.01-2.07) \\
0.25(0.10-0.36) \\
0.53(0.12-0.95)\end{array}$ & $\begin{array}{l}0.21(0.12-0.30) \\
0.23(0.13-0.41) \\
1.04(0.01-4.34) \\
0.53(0.25-1.12) \\
0.40(0.18-0.81)\end{array}$ & $\begin{array}{l}0.91(0.51-1.97) \\
3.61(0.64-7.46) \\
2.90(0.04-8.91) \\
1.73(0.51-3.41) \\
1.64(0.69-3.06)\end{array}$ & $\begin{array}{l}5 \\
5 \\
7 \\
5 \\
5\end{array}$ \\
\hline 6 & $\begin{array}{l}2: 1 \text { mixture } \\
\text { NPC }-40 \\
\text { NPH-50 } \\
\text { Protamine zinc } \\
\text { Globin with zinc }\end{array}$ & $\begin{array}{l}35 \\
35 \\
35 \\
35 \\
35\end{array}$ & $\begin{array}{l}1.36(0.76-1.96) \\
2.19(0.66-3.04) \\
1.70(0.82-3.27) \\
0.95(0.52-1.38) \\
2.12(1.39-2.52)\end{array}$ & $\begin{array}{l}0.24(0.12-0.35) \\
2.00(1.28-2.90) \\
1.72(0.76-2.58) \\
2.24(0.17-4.32) \\
1.17(0.96-1.59)\end{array}$ & $\begin{array}{l}0.48(0.08-0.89) \\
1.25(0.49-2.70) \\
1.34(0.89-1.47) \\
0.37(0.05-0.69) \\
1.33(0.74-1.88)\end{array}$ & $\begin{array}{l}0.28(0.22-0.33) \\
0.58(0.26-1.09) \\
0.67(0.40-0.91) \\
0.56(0.07-1.04) \\
0.43(0.27-0.73)\end{array}$ & $\begin{array}{l}2.36(2.33-2.38) \\
6.01(4.96-7.35) \\
5.50(3.96-7.45) \\
4.12(0.81-7.43) \\
5.06(3.37-6.20)\end{array}$ & $\begin{array}{l}2 \\
3 \\
3 \\
2 \\
3\end{array}$ \\
\hline 7 & $\begin{array}{l}2: 1 \text { mixture } \\
\text { NPC }-40 \\
\text { NPH-50 } \\
\text { Protamine zinc } \\
\text { Globin with zinc }\end{array}$ & $\begin{array}{l}26 \\
24 \\
23 \\
23 \\
24\end{array}$ & $\begin{array}{l}2.61(0.01-12.60) \\
0.27(0.07-0.53) \\
1.64(0.34-4.02) \\
2.05(0.39-5.00) \\
2.76(0.14-6.93)\end{array}$ & $\begin{array}{l}1.40(0.07-6.02) \\
0.43(0.07-0.93) \\
1.38(0.22-3.20) \\
4.18(1.04-14.00) \\
1.19(0.18-4.52)\end{array}$ & $\begin{array}{l}1.12(0.07-4.16) \\
0.39(0.07-0.83) \\
0.60(0.06-2.14) \\
3.00(0.98-7.22) \\
1.24(0.23-4.03)\end{array}$ & $\begin{array}{l}1.27(0.06-2.95) \\
0.70(0.02-1.46) \\
0.58(0.07-1.62) \\
1.98(0.13-7.45) \\
0.96(0.06-2.17)\end{array}$ & $\begin{array}{c}6.40(0.27-4.05) \\
1.80(0.23-3.75) \\
4.20(1.58-8.06) \\
11.22(3.16-23.28) \\
6.14(2.74-12.96)\end{array}$ & $\begin{array}{l}8 \\
4 \\
7 \\
7 \\
7\end{array}$ \\
\hline 9 & $\begin{array}{l}2: 1 \text { mixture } \\
\text { NPC }-40 \\
\text { NPH-50 } \\
\text { Protamine zinc } \\
\text { Globin with zinc }\end{array}$ & $\begin{array}{l}47 \\
40 \\
39 \\
36 \\
44\end{array}$ & $\begin{array}{l}1.04(0.34-2.06) \\
1.76(0.52-3.76) \\
0.40(0.03-1.55) \\
1.14(0.25-2.69) \\
1.22(0.27-3.81)\end{array}$ & $\begin{array}{l}0.48(0.16-1.58) \\
1.55(0.87-2.41) \\
0.52(0.03-1.56) \\
0.85(0.12-2.16) \\
1.64(0.04-5.00)\end{array}$ & $\begin{array}{l}1.26(0.18-5.49) \\
0.32(0.21-0.48) \\
0.31(0.03-0.93) \\
0.68(0.37-1.06) \\
0.24(0.01-0.79)\end{array}$ & $\begin{array}{l}1.52(0.27-5.07) \\
0.21(0.11-0.29) \\
0.37(0.04-0.92) \\
0.29(0.14-0.44) \\
0.28(0.01-0.61)\end{array}$ & $\begin{array}{l}4.04(0.54-13.50) \\
3.84(1.98-6.74) \\
1.60(0.13-3.46) \\
2.96(1.27-6.19) \\
3.39(1.48-9.29)\end{array}$ & $\begin{array}{r}6 \\
3 \\
10 \\
3 \\
7\end{array}$ \\
\hline
\end{tabular}


TABLE IV-Continued

\begin{tabular}{|c|c|c|c|c|c|c|c|c|}
\hline \multirow{2}{*}{ Patient } & \multirow{2}{*}{ Insulin } & \multirow{2}{*}{$\begin{array}{l}\text { Aver- } \\
\text { age } \\
\text { daily } \\
\text { dose }\end{array}$} & \multicolumn{4}{|c|}{ Mean and range of urinary sugar in $\mathrm{g}$. from } & \multirow{2}{*}{$\begin{array}{l}\text { Daily } \\
\text { total }\end{array}$} & \multirow{2}{*}{$\begin{array}{l}\text { No. of } \\
\text { days of } \\
\text { obser- } \\
\text { vation }\end{array}$} \\
\hline & & & $\begin{array}{l}\text { 7:30 a.m.- } \\
\text { 11:30 a.m. }\end{array}$ & $\begin{array}{r}11: 30 \text { a.m.- } \\
4: 30 \text { p.m. }\end{array}$ & $\begin{array}{l}\text { 4:30 p.m.- } \\
\text { 9:30 p.m. }\end{array}$ & $\begin{array}{l}\text { 9:30 p.m.- } \\
7: 30 \text { a.m. }\end{array}$ & & \\
\hline \multirow[t]{2}{*}{10} & \multirow{2}{*}{$\begin{array}{l}2: 1 \text { mixture } \\
\text { NPC }-40 \\
\text { NPH-50 } \\
\text { Protamine zinc } \\
\text { Globin with zinc }\end{array}$} & $\begin{array}{l}240 \\
220 \\
220\end{array}$ & $\begin{array}{l}1.48(0.72-2.69) \\
0.31(0.16-0.72) \\
1.27(0.02-3.91)\end{array}$ & $\begin{array}{l}1.73(0.31-4.02) \\
0.45(0.14-1.82) \\
1.06(0.02-3.16)\end{array}$ & $\begin{array}{l}1.28(0.36-2.98) \\
0.19(0.10-0.28) \\
0.32(0.02-1.31)\end{array}$ & $\begin{array}{l}1.14(0.42-1.56) \\
0.22(0.16-0.43) \\
0.72(0.04-3.86)\end{array}$ & $\begin{array}{l}8.11(1.94-15.55) \\
1.18(0.64-2.92) \\
3.36(0.10-8.62)\end{array}$ & $\begin{array}{l}3 \\
6 \\
8\end{array}$ \\
\hline & & 200 & $1.39(0.06-5.06)$ & $0.95(0.06-3.07)$ & $0.23(0.06-0.43)$ & $0.44(0.08-0.93)$ & $3.02(0.26-8.75)$ & 4 \\
\hline 11 & $\begin{array}{l}2: 1 \text { mixture } \\
\text { NPC-40 } \\
\text { NPH-50 } \\
\text { Protamine zinc } \\
\text { Globin with zinc }\end{array}$ & $\begin{array}{l}49 \\
49 \\
49 \\
42 \\
48\end{array}$ & $\begin{array}{l}3.70(0.20-15.10) \\
2.12(0.10-3.00) \\
3.72(1.70-9.50) \\
3.24(0.20-8.00) \\
5.12(2.10-10.00)\end{array}$ & $\begin{array}{l}5.56(0.30-15.10) \\
1.08(0.30-1.90) \\
1.87(0.10-5.90) \\
6.80(1.00-13.90) \\
0.63(0.10-1.10)\end{array}$ & $\begin{array}{l}2.06(0.00-10.20) \\
2.04(0.30-9.80) \\
0.98(0.20-2.40) \\
4.62(0.50-12.20) \\
1.00(0.10-3.30)\end{array}$ & $\begin{array}{l}0.94(0.10-7.60) \\
0.69(0.10-3.80) \\
0.50(0.20-1.60) \\
1.30(0.20-3.60) \\
2.21(0.20-9.50)\end{array}$ & \begin{tabular}{|}
$12.26(2.30-37.50)$ \\
$5.93(2.80-16.40)$ \\
$7.07(3.80-11.20)$ \\
$15.96(3.30-30.90)$ \\
$10.33(5.40-18.50)$
\end{tabular} & $\begin{array}{r}13 \\
6 \\
6 \\
5 \\
6\end{array}$ \\
\hline 12 & $\begin{array}{l}2: 1 \text { mixture } \\
\text { NPC }-40 \\
\text { NPH-50 } \\
\text { Protamine zinc } \\
\text { Globin with zinc }\end{array}$ & $\begin{array}{l}30 \\
30 \\
30 \\
28 \\
30\end{array}$ & $\begin{array}{l}0.56(0.10-1.30) \\
1.10(0.14-3.09) \\
1.14(0.03-3.78) \\
3.57(1.15-6.85) \\
1.61(0.20-5.15)\end{array}$ & $\begin{array}{l}1.40(0.10-2.74) \\
1.56(0.14-6.84) \\
2.38(0.03-7.20) \\
4.26(0.58-9.80) \\
0.53(0.17-1.00)\end{array}$ & $\begin{array}{l}0.25(0.13-0.35) \\
0.82(0.14-3.16) \\
2.01(0.02-6.20) \\
3.30(1.30-7.70) \\
1.41(0.15-4.40)\end{array}$ & $\begin{array}{l}0.36(0.14-0.52) \\
1.22(0.12-2.59) \\
0.72(0.02-2.42) \\
1.50(0.25-4.80) \\
1.46(0.11-5.30)\end{array}$ & \begin{tabular}{|c}
$2.57(0.47-3.82)$ \\
$4.70(0.54-13.08)$ \\
$6.26(0.10-19.60)$ \\
$12.63(4.02-29.15)$ \\
$5.02(1.55-10.07)$
\end{tabular} & $\begin{array}{l}3 \\
3 \\
7 \\
4 \\
4\end{array}$ \\
\hline 13 & $\begin{array}{l}2: 1 \text { mixture } \\
\text { NPC-40 } \\
\text { NPH -50 } \\
\text { Protamine zinc } \\
\text { Globin with zinc }\end{array}$ & $\begin{array}{l}65 \\
50 \\
55 \\
45 \\
40\end{array}$ & $\begin{array}{l}1.17(0.56-1.88) \\
2.24(0.25-3.14) \\
1.91(0.20-5.43) \\
6.05(0.33-16.10) \\
1.46(0.42-3.12)\end{array}$ & $\begin{array}{l}2.11(0.59-3.12) \\
1.64(0.21-2.92) \\
1.62(0.06-3.74) \\
4.47(0.17-11.41) \\
1.56(0.06-3.10)\end{array}$ & $\begin{array}{l}1.34(0.45-2.58) \\
0.68(0.21-1.42) \\
0.68(0.02-3.25) \\
7.13(0.21-15.12) \\
0.52(0.01-1.11)\end{array}$ & $\begin{array}{l}3.85(2.38-6.55) \\
1.84(0.10-5.70) \\
1.34(0.05-4.86) \\
6.94(0.22-14.00) \\
0.76(0.11-2.51)\end{array}$ & $\begin{array}{c}8.46(5.86-12.67) \\
6.40(1.22-1.41) \\
5.56(1.78-13.69) \\
21.63(3.67-49.55) \\
4.30(0.93-7.80)\end{array}$ & $\begin{array}{r}4 \\
7 \\
13 \\
7 \\
8\end{array}$ \\
\hline \multirow[t]{2}{*}{14} & \multirow{2}{*}{$\begin{array}{l}2: 1 \text { mixture } \\
\text { NPC-40 } \\
\text { NPH-50 } \\
\text { Protamine zinc } \\
\text { Globin with zinc }\end{array}$} & $\begin{array}{l}54 \\
55 \\
55\end{array}$ & $\begin{array}{l}1.94(0.04-6.13) \\
2.85(0.08-11.35) \\
5.42(0.09-12.04)\end{array}$ & $\begin{array}{l}2.20(0.05-11.65) \\
1.56(0.06-4.14) \\
5.02(0.04-14.63)\end{array}$ & $\begin{array}{l}0.71(0.03-6.30) \\
0.52(0.10-2.14) \\
3.77(0.28-7.64)\end{array}$ & $\begin{array}{l}0.60(0.03-3.24) \\
0.25(0.10-0.40) \\
5.38(0.33-21.80)\end{array}$ & $\begin{array}{r}5.74(0.22-22.00) \\
5.18(0.62-16.11) \\
19.59(2.18-33.20)\end{array}$ & $\begin{array}{r}15 \\
8 \\
9\end{array}$ \\
\hline & & 56 & $4.71(0.03-13.54)$ & $2.06(0.03-8.88)$ & $0.22(0.03-0.37)$ & $3.62(0.11-17.96)$ & $10.60(0.42-22.88)$ & 6 \\
\hline 15 & No data & & & & & & & \\
\hline 16 & $\begin{array}{l}2: 1 \text { mixture } \\
\text { NPC }-40 \\
\text { NPH-50 } \\
\text { Protamine zinc } \\
\text { Globin with zinc }\end{array}$ & $\begin{array}{l}75 \\
55 \\
61 \\
49 \\
55\end{array}$ & $\begin{array}{l}2.88(0.17-8.65) \\
0.84(0.14-1.88) \\
4.11(0.20-14.99) \\
2.70(0.15-8.16) \\
2.69(0.42-4.87)\end{array}$ & $\begin{array}{l}4.42(0.22-11.88) \\
2.48(0.40-7.18) \\
2.02(0.16-5.67) \\
7.58(0.06-24.65) \\
1.82(0.26-6.26)\end{array}$ & $\begin{array}{l}2.70(0.24-13.30) \\
2.38(0.77-5.17) \\
3.34(0.05-10.60) \\
6.80(0.17-13.35) \\
3.36(0.25-12.80)\end{array}$ & $\begin{array}{l}3.66(0.08-14.60) \\
7.19(0.39-255.00) \\
4.66(0.24-11.65) \\
3.69(0.39-6.36) \\
1.42(0.88-1.87)\end{array}$ & $\begin{array}{c}13.65(1.81-48.15) \\
11.41(2.17-33.21) \\
13.94(1.05-29.94) \\
20.77(10.60-46.16) \\
9.29(4.86-21.50)\end{array}$ & $\begin{array}{l}6 \\
4 \\
8 \\
4 \\
5\end{array}$ \\
\hline \multirow[t]{2}{*}{17} & \multirow{2}{*}{$\begin{array}{l}2: 1 \text { mixture } \\
\text { NPC }-40 \\
\text { NPH-50 } \\
\text { Protamine zinc } \\
\text { Globin with zinc }\end{array}$} & $\begin{array}{l}48 \\
50 \\
52\end{array}$ & $\begin{array}{l}0.81(0.04-2.58) \\
1.87(0.06-5.39) \\
1.90(0.03-3.62)\end{array}$ & $\begin{array}{l}0.59(0.04-2.03) \\
2.40(0.06-8.20) \\
3.98(0.03-9.52)\end{array}$ & $\begin{array}{l}0.30(0.04-0.96) \\
3.10(1.06-20.75) \\
1.42(0.03-3.52)\end{array}$ & $\begin{array}{l}1.30(0.04-4.95) \\
5.64(0.08-31.00) \\
3.42(0.02-7.41)\end{array}$ & $\begin{array}{r}7.70(0.16-27.65) \\
13.02(0.26-38.35) \\
10.73(0.11-20.88)\end{array}$ & $\begin{array}{r}4 \\
10 \\
4\end{array}$ \\
\hline & & 48 & $9.22(0.05-15.20)$ & $3.43(0.92-7.10)$ & $1.15(0.13-4.04)$ & $2.46(0.58-4.70)$ & $16.25(5.92-24.62)$ & 5 \\
\hline \multirow[t]{2}{*}{18} & \multirow{2}{*}{\begin{tabular}{|l|}
$2: 1$ mixture \\
NPC-40 \\
NPH-50 \\
Protamine zinc \\
Globin with zinc
\end{tabular}} & $\begin{array}{l}30 \\
32 \\
33\end{array}$ & $\begin{array}{l}8.14(0.12-16.40) \\
8.07(0.03-17.20) \\
2.47(0.04-9.80)\end{array}$ & $\begin{array}{l}1.34(0.07-7.55) \\
5.96(0.02-20.60) \\
1.45(0.05-7.90)\end{array}$ & $\begin{array}{l}0.82(0.07-4.00) \\
5.04(0.07-12.00) \\
4.12(0.10-20.21)\end{array}$ & $\begin{array}{l}6.58(0.03-38.10) \\
4.30(0.01-17.40) \\
5.12(0.11-17.98)\end{array}$ & $\begin{array}{l}16.88(5.38-48.98) \\
23.37(0.13-63.10) \\
13.16(0.38-47.01)\end{array}$ & $\begin{array}{l}8 \\
7 \\
6\end{array}$ \\
\hline & & 34 & $8.04(0.07-21.70)$ & $1.80(0.21-3.90)$ & $1.65(0.17-4.60)$ & $6.69(0.05-16.72)$ & $18.18(0.50-27.02)$ & 4 \\
\hline \multirow[t]{2}{*}{19} & \multirow{2}{*}{\begin{tabular}{|l|}
$2: 1$ mixture \\
NPC -40 \\
NPH-50 \\
Protamine zinc \\
Globin with zinc
\end{tabular}} & $\begin{array}{l}51 \\
57 \\
48\end{array}$ & $\begin{array}{r}7.98(1.97-17.00) \\
14.33(7.50-25.80) \\
5.27(0.94-20.70)\end{array}$ & $\begin{array}{c}13.04(4.54-24.20) \\
23.67(15.00-40.40) \\
3.68(0.11-9.15)\end{array}$ & $\begin{array}{c}8.38(0.44-23.00) \\
10.55(4.60-13.70) \\
2.50(0.79-5.75)\end{array}$ & $\begin{array}{c}9.56(0.77-21.00) \\
16.30(4.60-31.10) \\
3.34(0.28-9.35)\end{array}$ & $\begin{array}{l}38.96(8.32-56.65) \\
54.97(24.32-75.40) \\
16.06(3.63-36.45)\end{array}$ & $\begin{array}{l}8 \\
3 \\
8\end{array}$ \\
\hline & & 44 & $8.54(5.64-13.00)$ & $7.54(0.13-14.80)$ & $2.97(0.56-5.72)$ & $3.01(0.87-5.25)$ & $20.21(12.77-34.39)$ & 4 \\
\hline
\end{tabular}

encountered had negligible effects on any of the measures of response used. The validity of this assumption is supported by the lack of any apparent relation when any of the measures of response is plotted against unit dose. It has also been necessary to assume that the changes in diet distribution which were necessary in some of the patients had, at worst, a leveling effect on the quantities investigated. Hence, when differences associated with kind of insulin are observed it is felt that they would be larger rather than smaller if diet distribution had been entirely uniform over all insulins for each patient.

\section{RESULTS}

Intra-daily variation. On the present measurement scale intra-daily variation in blood sugar ranges from about 2.00 in non-diabetics to about 5.00 for the highest value obtained in this investigation. The value for non-diabetics was calculated from published data (37). Although these data are not directly comparable with those of the present study, the value so obtained provides an approximate normal level for intra-daily variation in blood sugar.

Table $\mathrm{V}$ presents the average values for intradaily variation in blood sugar for each patient on each insulin. In the eight patients marked with the asterisk the probability of obtaining the observed results on the hypothesis that the true intra-daily variation is the same with each of the insulins tested is less than 5\%. In these patients, then, it is decided that there are changes in the 
level of intra-daily variation associated with changes in kind of insulin administered. The differences observed in the other patients are small enough to have arisen easily by chance. Although individual patients show different levels of intradaily variation in blood sugar on the different insulins, there is little consistency from one patient to another. The data of this study do not demonstrate any tendency for one insulin to maintain this measure at lower levels than another when results are considered over all patients.

From the definition of intra-daily variation in urinary sugar, it is clear that this quantity takes the value zero when there is no glycosuria. The highest value obtained in the present investigation was approximately 2.50 . Table VI presents the average values of the intra-daily variation in urinary sugar. Again the patients in which the differences among insulins are too large to be reasonably ascribed to sampling variation have been marked with the asterisk. No tendency is apparent for any one insulin to maintain intra-daily variation in urinary sugar at levels higher or lower than any other insulin.

Inter-daily variation. The highest value of

TABLE V

Average intra-daily variation in blood sugar

\begin{tabular}{c|c|c|c|c|c}
\hline \hline $\begin{array}{c}\text { Patient } \\
\text { number }\end{array}$ & $\begin{array}{c}2: 1 \\
\text { mixture }\end{array}$ & $\begin{array}{c}\text { NPC-40 } \\
\text { insulin }\end{array}$ & $\begin{array}{c}\text { NPH-50 } \\
\text { insulin }\end{array}$ & $\begin{array}{c}\text { Protamine } \\
\text { zinc } \\
\text { insulin }\end{array}$ & $\begin{array}{c}\text { Globin } \\
\text { insulin } \\
\text { with } \\
\text { zinc }\end{array}$ \\
\hline 1 & $2.53(3) \dagger$ & $2.61(3)$ & $2.75(6)$ & $3.09(5)$ & $2.88(3)$ \\
$2^{*}$ & $3.06(10)$ & $3.22(9)$ & $3.44(11)$ & $3.41(6)$ & $2.95(5)$ \\
$3^{*}$ & $3.42(4)$ & $3.42(4)$ & $2.57(4)$ & $3.01(4)$ & $3.17(4)$ \\
4 & $3.16(3)$ & $3.20(3)$ & $3.59(5)$ & $3.99(3)$ & $3.70(3)$ \\
5 & $3.32(5)$ & $3.76(5)$ & $3.54(8)$ & $3.52(5)$ & $3.21(5)$ \\
6 & $3.56(2)$ & $3.16(3)$ & $3.18(2)$ & $2.99(3)$ & $2.99(2)$ \\
7 & $3.57(8)$ & $3.74(3)$ & $3.61(7)$ & $3.91(7)$ & $3.50(7)$ \\
8 & $3.77(4)$ & $3.66(4)$ & $3.66(8)$ & $3.84(4)$ & $3.77(8)$ \\
$9^{*}$ & $3.65(7)$ & $3.80(3)$ & $3.47(10)$ & $3.50(2)$ & $4.06(7)$ \\
$10^{*}$ & $3.63(3)$ & $3.56(4)$ & $3.23(8)$ & & $3.89(4)$ \\
11 & $3.66(10)$ & $3.26(5)$ & $3.72(5)$ & $3.96(4)$ & $3.57(5)$ \\
$12^{*}$ & $3.33(3)$ & $3.39(3)$ & $3.47(7)$ & $4.30(4)$ & $3.45(4)$ \\
13 & $3.97(4)$ & $3.54(8)$ & $3.59(12)$ & $3.77(8)$ & $3.97(8)$ \\
$14^{*}$ & $3.75(13)$ & $3.71(7)$ & $4.28(9)$ & & $4.39(5)$ \\
15 & $4.14(13)$ & $4.50(4)$ & $4.00(4)$ & & $4.13(3)$ \\
16 & $4.02(6)$ & $4.01(5)$ & $3.92(8)$ & $3.66(4)$ & $4.24(5)$ \\
$17^{*}$ & $3.35(5)$ & $4.04(9)$ & $3.75(5)$ & & $4.94(5)$ \\
$18^{*}$ & $4.24(7)$ & $4.05(6)$ & $3.39(5)$ & & $4.34(3)$ \\
19 & $4.37(8)$ & $4.34(4)$ & $4.06(8)$ & & $4.47(5)$ \\
\hline
\end{tabular}

* The patients marked with the asterisk are those showing differences among the insulins which are significant at the $5 \%$ level.

$t$ The numbers in the parentheses are values of $n$, the number of days of observation on which the average is based. The standard error of any average in the table is approximately equal to $\left(1 / v_{\mathbf{n}}\right)(.435)$.
TABLE VI

Average intra-daily variation in urine sugar

\begin{tabular}{c|c|c|c|c|c|c}
\hline \hline $\begin{array}{c}\text { Patient } \\
\text { number }\end{array}$ & $\begin{array}{c}2: 1 \\
\text { mixture }\end{array}$ & $\begin{array}{c}\text { NPC-40 } \\
\text { insulin }\end{array}$ & $\begin{array}{c}\text { NPH-50 } \\
\text { insulin }\end{array}$ & $\begin{array}{c}\text { Prota- } \\
\text { mine } \\
\text { zinc } \\
\text { insulin }\end{array}$ & $\begin{array}{c}\text { Globin } \\
\text { insulin } \\
\text { with } \\
\text { zinc }\end{array}$ \\
\hline 1 & $0.00(3)+$ & $0.00(3)$ & $0.11(6)$ & $0.03(5)$ & $0.00(3)$ \\
2 & $0.04(12)$ & $0.07(8)$ & $0.05(13)$ & $0.01(6)$ & $0.03(6)$ \\
3 & 0.01 & $(3)$ & $0.00(4)$ & $0.01(4)$ & $0.08(4)$ & $0.02(4)$ \\
4 & 0.01 & $(2)$ & $0.05(3)$ & $0.29(6)$ & $0.27(3)$ & $0.27(3)$ \\
$5^{*}$ & 0.03 & $(4)$ & $0.58(5)$ & $0.34(7)$ & $0.06(5)$ & $0.04(5)$ \\
6 & 0.32 & $(2)$ & $0.72(3)$ & $0.41(3)$ & $0.51(2)$ & $0.41(3)$ \\
$7^{*}$ & 0.56 & $(8)$ & $0.07(4)$ & $0.53(7)$ & $0.89(7)$ & $0.84(7)$ \\
8 & $0.30(3)$ & $0.02(1)$ & $0.30(10)$ & $0.70(4)$ & $0.32(8)$ \\
9 & $0.48(6)$ & $0.47(3)$ & $0.10(10)$ & $0.24(3)$ & $0.51(7)$ \\
10 & $0.28(3)$ & $0.08(6)$ & $0.37(8)$ & & $0.33(4)$ \\
11 & 1.31 & $(13)$ & $0.86(6)$ & $0.99(6)$ & $1.43(5)$ & $1.33(6)$ \\
12 & $0.33(3)$ & $0.45(6)$ & $0.43(4)$ & $0.83(4)$ & $0.69(4)$ \\
13 & 0.54 & $(4)$ & $0.58(7)$ & $0.66(13)$ & $1.11(7)$ & $0.40(8)$ \\
$14^{*}$ & 0.77 & $(15)$ & $0.60(8)$ & $1.63(9)$ & & $1.28(6)$ \\
15 & & & & & & \\
16 & 1.00 & $(6)$ & $1.08(4)$ & $1.28(8)$ & $1.76(4)$ & $1.11(5)$ \\
$17^{*}$ & $0.26(4)$ & $1.06(10)$ & $0.68(4)$ & & $1.70(5)$ \\
$18^{*}$ & $1.83(8)$ & $1.23(7)$ & $1.03(6)$ & & $1.64(4)$ \\
$19^{*}$ & $1.86(8)$ & $2.32(3)$ & $1.32(8)$ & & $1.65(4)$ \\
\hline
\end{tabular}

* The patients marked with the asterisk are those showing significant differences among insulins.

$\downarrow$ The numbers in the parentheses are values of $n$, the number of days on which the average is based. The standard error of any value in the table is approximately $\left(1 / v_{n}\right)(.303)$ for patients $1-7$ and $\left(1 / v_{\bar{n}}\right)(.596)$ for patients 8-19.

inter-daily variation in blood sugar obtained here was about 5.50. No direct estimate of this quantity was available for non-diabetics. Calculated "guesses" based on published data (37) in which non-diabetics were observed for just one day place the figure in the neighborhood of 2.50 . Inter-daily variation in urinary sugar ranges from zero in individuals showing no glycosuria to about 3.00 for the highest value in this study.

Table VII presents the average values for interdaily variation in blood sugar. In no case are the differences associated with insulins sufficiently large to indicate anything except sampling variation. Average values of inter-daily variation in urinary sugar are shown in Table VIII. In this respect there also seems to be no real difference among insulins.

Mean deviations from the daily mean. The results of this part of the analysis are presented graphically in Figures 1-5, each Figure corresponding to one kind of insulin. Part $A$ in each Figure plots the results for the first seven or stable patients while Part B plots the results for the last twelve or unstable patients. It may be noted that each line in these figures represents the 
TABLE VII

Average inter-daily variation in blood sugar

\begin{tabular}{c|c|c|c|c|c}
\hline \hline $\begin{array}{c}\text { Patient } \\
\text { number }\end{array}$ & $\begin{array}{c}2: 1 \\
\text { mixture }\end{array}$ & $\begin{array}{c}\text { NPC-40 } \\
\text { insulin }\end{array}$ & $\begin{array}{c}\text { NPH-50 } \\
\text { insulin }\end{array}$ & $\begin{array}{c}\text { Prota- } \\
\text { mine } \\
\text { zinc } \\
\text { insulin }\end{array}$ & $\begin{array}{c}\text { Globin } \\
\text { insulin } \\
\text { with } \\
\text { zinc }\end{array}$ \\
\hline 1 & $3.26(1) *+$ & $3.35(1)$ & $3.02(2)$ & $3.65(1)$ & $2.38(1)$ \\
2 & $3.82(2)$ & $3.68(2)$ & $3.39(3)$ & $3.28(1)$ & $3.61(1)$ \\
3 & $3.58(1)$ & $3.18(1)$ & $3.61(1)$ & $4.27(1)$ & $3.44(1)$ \\
4 & $3.90(1)$ & $3.12(1)$ & $3.89(1)$ & $4.12(1)$ & $3.74(1)$ \\
5 & $2.81(1)$ & $3.90(1)$ & $3.46(1)$ & $3.47(1)$ & $3.41(1)$ \\
6 & $3.95(1)$ & $3.95(1)$ & $3.35(1)$ & $4.17(1)$ & $3.73(1)$ \\
7 & $3.82(2)$ & $3.71(1)$ & $3.59(2)$ & $3.95(2)$ & $3.47(2)$ \\
8 & $3.98(1)$ & $4.20(1)$ & $4.21(2)$ & $4.70(2)$ & $3.92(1)$ \\
9 & $4.26(2)$ & $4.15(1)$ & $3.52(3)$ & $4.56(1)$ & $4.06(2)$ \\
10 & $4.92(1)$ & $3.06(1)$ & $4.16(2)$ & & $4.40(1)$ \\
11 & $4.08(2)$ & $4.23(1)$ & $3.91(2)$ & & $3.46(1)$ \\
12 & $3.94(1)$ & $4.43(1)$ & $4.54(2)$ & $4.45(1)$ & $4.09(1)$ \\
13 & $3.82(1)$ & $4.27(2)$ & $3.58(5)$ & $4.38(2)$ & $4.17(2)$ \\
14 & $4.10(5)$ & $4.28(1)$ & $4.59(2)$ & & $4.77(1)$ \\
15 & $4.34(3)$ & $4.12(1)$ & $4.74(1)$ & & $3.65(1)$ \\
16 & $4.54(1)$ & $4.55(1)$ & $4.94(2)$ & $4.60(1)$ & $4.56(1)$ \\
17 & $4.30(1)$ & $4.93(2)$ & $5.38(1)$ & & $4.42(1)$ \\
18 & $4.53(2)$ & $5.15(2)$ & $4.61(2)$ & & $4.90(1)$ \\
19 & $4.49(3)$ & $3.91(1)$ & $3.82(3)$ & & $4.75(1)$ \\
\hline
\end{tabular}

* The numbers in the parentheses are values of $p$, the number of "groups" of days on which the average is based. The standard error of any average in the table is approximately equal to $\left(1 / v_{\bar{p}}\right)(.565)$.

$t$ The standard error in this case depends also upon the number of days in each "group" involved. Since the numbers of days in the "groups" did not vary excessively about the mode of 3 , this factor has been ignored.

average over all the days on which the patient received the given insulin. If the lines were plotted separately for each day the high variability in the

TABLE VIII

Average inter-daily variation in urine sugar

\begin{tabular}{r|c|c|c|c|c}
\hline \hline $\begin{array}{c}\text { Patient } \\
\text { number }\end{array}$ & $\begin{array}{c}2: 1 \\
\text { mixture }\end{array}$ & $\begin{array}{c}\text { NPC-40 } \\
\text { insulin }\end{array}$ & $\begin{array}{c}\text { NPH-50 } \\
\text { insulin }\end{array}$ & $\begin{array}{c}\text { Prota- } \\
\text { mine } \\
\text { zinc } \\
\text { insulin }\end{array}$ & $\begin{array}{c}\text { Globin } \\
\text { insulin } \\
\text { with } \\
\text { zinc }\end{array}$ \\
\hline 1 & $0.00(1) *$ & $0.02(1)$ & $0.61(2)$ & $0.13(1)$ & $0.07(1)$ \\
2 & $0.52(3)$ & $0.22(2)$ & $0.04(3)$ & $0.02(1)$ & $0.04(1)$ \\
3 & $0.04(1)$ & $0.02(1)$ & $0.01(1)$ & $0.05(1)$ & $0.06(1)$ \\
4 & $0.11(1)$ & $0.19(1)$ & $0.82(2)$ & $0.82(1)$ & $0.79(1)$ \\
5 & $0.13(1)$ & $1.06(1)$ & $0.54(1)$ & $0.18(1)$ & $0.32(1)$ \\
6 & $0.00(1)$ & $0.40(1)$ & $0.63(1)$ & $1.36(1)$ & $0.51(1)$ \\
7 & $1.08(2)$ & $0.61(1)$ & $0.52(2)$ & $1.15(2)$ & $0.86(2)$ \\
8 & $0.77(1)$ & & $0.69(3)$ & $1.43(1)$ & $0.82(2)$ \\
9 & $0.91(2)$ & $0.87(1)$ & $0.12(3)$ & $0.94(1)$ & $0.55(2)$ \\
10 & $1.40(1)$ & $0.18(2)$ & $0.64(2)$ & & $1.21(1)$ \\
11 & $1.97(3)$ & $1.57(1)$ & $1.15(2)$ & $1.76(1)$ & $1.27(1)$ \\
12 & $0.64(1)$ & $1.38(1)$ & $1.93(1)$ & $2.23(1)$ & $1.24(1)$ \\
13 & $1.05(1)$ & $0.97(3)$ & $0.52(5)$ & $1.14(2)$ & $0.84(2)$ \\
14 & $1.01(5)$ & $0.99(1)$ & $1.64(2)$ & & $1.92(1)$ \\
15 & & & & & \\
16 & $2.49(1)$ & $2.33(1)$ & $2.03(1)$ & $0.50(1)$ & $1.68(1)$ \\
17 & $1.42(1)$ & $1.89(2)$ & $2.14(1)$ & & $1.78(1)$ \\
18 & $1.68(3)$ & $2.41(2)$ & $1.66(2)$ & & $2.18(1)$ \\
19 & $2.16(3)$ & $2.51(1)$ & $1.95(3)$ & & $2.98(1)$ \\
\hline
\end{tabular}

* The numbers in the parentheses are values of $p$, the number of "groups" on which the average is based. shape from one day to another on the same insulin would be apparent. In spite of this large variability, however, statistical analysis supports the view that the consistency of the pattern is not a chance effect and that the apparent differences in pattern shown in Figures 1-5 are real differences. This consistency in pattern not only shows itself during any two days from the same period on a given insulin, but also in any two days from different periods on the same insulin.

In the stable group (Part $\mathrm{A}$ of Figures 1-5) the patterns for the different insulins are essentially the same. The three modifications of standard protamine zinc insulin produce patterns in the unstable group (Part B of Figures 1, 2 and 3), which are not only indistinguishable from one another but also similar to the patterns of the stable group. On the other hand, globin insulin with zinc and standard protamine zinc insulin produce patterns in the unstable group which are strikingly different from those of the other three insulins. The pattern of globin insulin with zinc displays a consistent tendency toward high points at 7:30 a.m. and 11:30 a.m. and low points at 4:30 p.m.

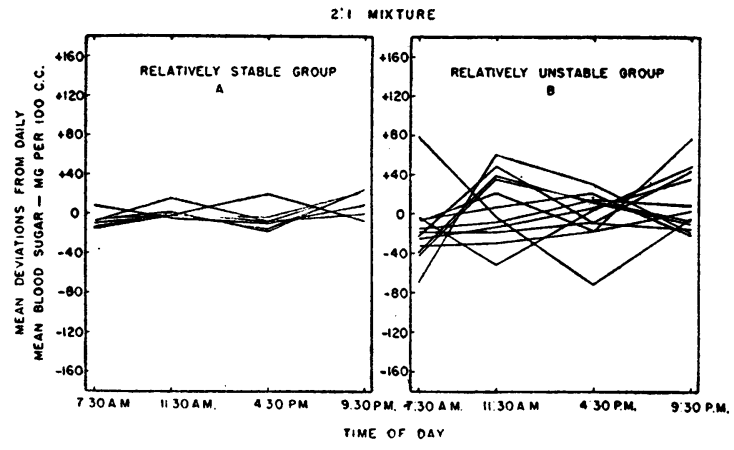

FIG. 1

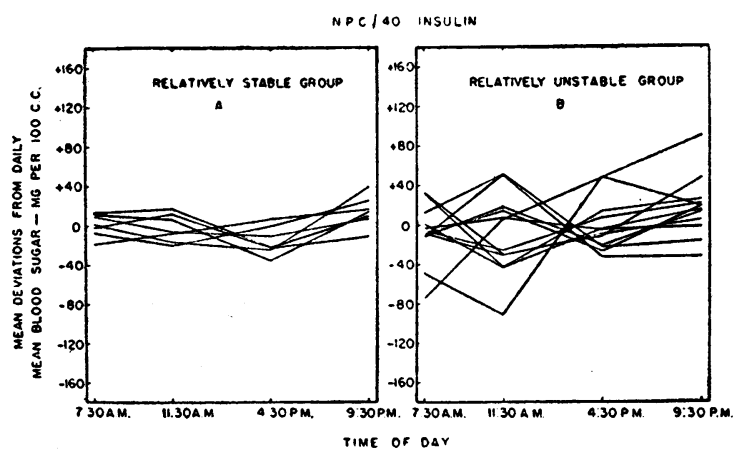

Fig. 2 


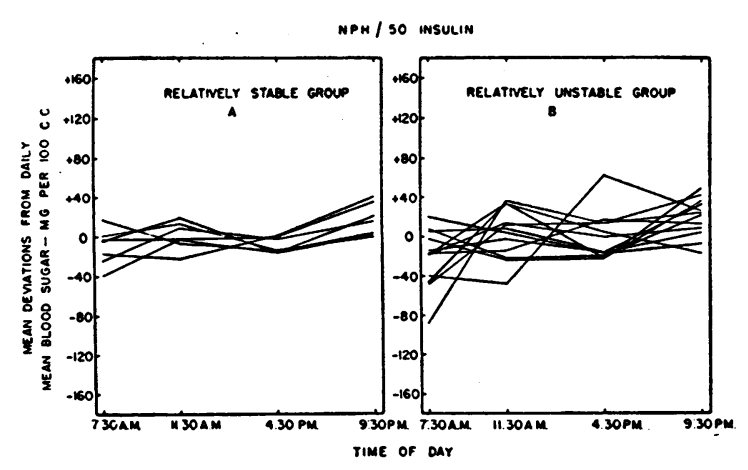

FIG. 3

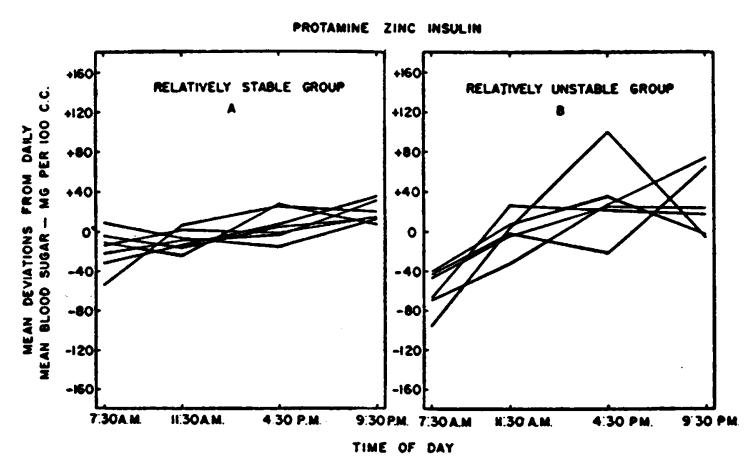

FIG. 4

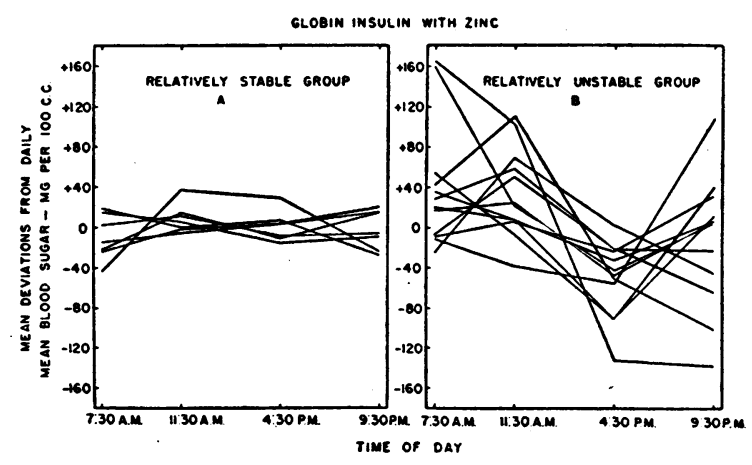

FIG. 5

and 9:30 p.m. (Part B of Figure 5). In contrast the pattern of standard protamine zinc insulin displays a consistent tendency toward low points at 7:30 a.m. and high points at $4: 30$ p.m. and 9:30 p. m. (Part B of Figure 4).$^{8}$ Both of these patterns should be contrasted with the more nearly level patterns in the other instances.

\footnotetext{
${ }^{8}$ It should be pointed out that the pattern might have been even more striking had it been possible to include all of the unstable group.
}

\section{DISCUSSION}

It is pertinent at this point to show the extent of agreement between the clinical classification of the patients and a classification based on the objective measures of response. The patients were ranked on the basis of each of the following quantities averaged over the whole period of study:

1.) Intra-daily variation in blood sugar

2.) Inter-daily variation in blood sugar

3.) Intra-daily variation in urine sugar

4.) Inter-daily variation in urine sugar

The agreement between the rankings on each of these bases although by no means perfect was found to be remarkably close. With one exception each of the rankings separately classified the patients into the stable and unstable groups in exactly the same way as clinical impression. The numbering of the patients in this paper is based on their respective average ranks. As would be expected no sharp line exists between the stable and unstable patients no matter what criterion of stability is used. The decision to make the break after seven patients was quite arbitrary. Be that as it may, some scale of stability seems desirable and meaningful. It is largely a matter of convenience in discussion to choose some dividing point on it. The essential conclusions would not be altered by moving this dividing point up or down by small amounts.

The two clinical groups which have been distinguished here on the basis of clinical impression and objective measures of variability possess the same characteristics as the two groups distinguished by Himsworth and Kerr (38) and Falta and colleagues (39) on the basis of sensitivity to insulin. Himsworth recognized two clinical groups of patients corresponding to the two types of diabetics differentiated by an insulin-glucose test. He remarked, "on the whole the insulin-sensitive diabetics tend to be younger, thin, to have a normal blood pressure and healthy arteries; in them the disease is sudden and severe at onset; they easily develop ketosis and react to a slight excess of insulin with a hypoglycemic attack. The insulininsensitive diabetics on the other hand tend to be older, to have hypertension and to exhibit arteriosclerosis; the onset of the disease is insidious; they rarely develop ketosis and can tolerate over-dosage 
of insulin without showing symptoms of hypoglycemia." It should be pointed out that Himsworth's description of insulin-sensitive and insulininsensitive diabetics describes exactly the unstable and stable diabetics respectively.

It would thus seem that generally speaking the stable diabetic is relatively insensitive to insulin whereas the unstable diabetic is relatively sensitive to insulin. However, a given patient's sensitivity to insulin fluctuates. At one time a given dose may invoke a profound hypoglycemia and yet the same dose may have little effect at another time. This is particularly true in the unstable patient and may give some insight into the occasional lack of agreement between the laboratory tests and clinical grouping of patients noted by both Himsworth and Falta. The general classification on the basis of stability is therefore considered preferable. It might be added that the so-called "brittle" diabetic $(40,41)$ is merely an example of marked instability.

Since the patients studied are not a random sample of the diabetic population, they provide no estimate of the true proportion of unstable diabetics. The series is biased in favor of the unstable group because it is felt that these are the patients in whom insulin timing is more critical. Some idea of the proportion of relatively unstable patients in the general diabetic population may be gleaned from the fact that in our diabetic clinic with a population between 250 and 300 the unstable group comprises probably not more than $25 \%$ of the entire group.

The importance of stability in the comparison of behavior of the different insulins is evident from the data presented. When the insulins are compared on their ability to control intra- and inter-daily variability, no large differences appear. Since intra-and inter-daily variations are measures of instability it becomes apparent that the ability of the insulins tested to affect stability is negligible. In spite of the best possible control of extraneous factors (diet, environment, and insulin) a certain basic instability remains, the degree of which is characteristic for each patient.

In contrast with extent of intra-daily variability, the pattern of intra-daily variability is affected differently by the insulins, though notable only in the unstable group of patients. In the stable group no serious timing defects are noted for any of the insulins studied. The patterns of distribution of insulin activity as described by the mean deviations from the daily mean of the blood sugar, at the times selected, are nearly level. The patterns are not only remarkably comparable from patient to patient, but even from insulin to insulin. For all practical purposes none of the insulins studied possesses any significant advantage over another in this group.

In the unstable group timing defects are brought into sharp focus. Globin insulin with zinc displays a consistent tendency to produce hypoglycemia in the late afternoon or evening. At the same time its action is too weak overnight to control adequately the morning fasting levels. The so-called "over-lapping" effect from dose to dose which is considered important in controlling post-breakfast hyperglycemia and glycosuria is either feeble or lacking altogether. The action of standard protamine zinc insulin is, in certain respects, opposite to that of globin insulin with zinc. Its action is too weak to control adequately post-prandial hyperglycemia and glycosuria. At the same time it has a distinct tendency to produce hypoglycemia overnight. In other words, a fault common to both insulins is too much activity at one time and too little at another. In contrast, the three modifications of standard protamine zinc insulin appear to have a more balanced distribution of insulin activity over a 24 hour period.

It is noteworthy that reactions to insulin were fewer, milder or less precipitous with any of the three modifications of standard protamine zinc insulin than with globin insulin with zinc or standard protamine zinc insulin. Also of interest is the observation that subcutaneous injections of any of the protamine insulins studied were decidedly less apt to cause local discomfort (immediate "burning" or "stinging" pain) than those of globin insulin with zinc.

Observations on the behaviour of the insulins in question have been continued in each of the 19 patients under their routine living conditions following discharge from the hospital. They have been followed at regular intervals (two to four weeks) through the facilities of the out-patient department for periods extending to as much as three years. Although the data obtained from 
this "follow-up study" do not permit quantitative comparisons, the general pattern of rèsponse to the different insulins is essentially the same as that already described. As a matter of fact, the increased physical activity of the patients incidental to pursuing their routine way of life has tended, if anything, to accentuate the timing defects. Finally, clinical experience with the insulins in question in over 100 patients followed on an out-patient status substantiates the opinions which have been formulated on the basis of the controlled studies presented.

The striking discrepancy in the performance of globin insulin with zinc and standard protamine zinc insulin in the two groups may be connected with the problem of homeostasis. In the stable patient any inherent defect in timing of the given insulin may be compensated for or minimized by homeostatic mechanisms which seem to be functioning to some extent; while in the unstable patient in whom the homeostatic mechanisms appear to be seriously disrupted and insulin timing more critical, any inherent faults stand out glaringly. For this reason comparison of time-action curves of various insulins in stable patients or in normal laboratory animals (31-33) cannot be strictly correlated with comparison of clinical responses to the same insulins. Difference in timing tends to be minimized in stable patients and accentuated in unstable patients. Some insight into the nature and mechanism of the observed defects of globin insulin with zinc and standard protamine zinc insulin is afforded by some nephelometric.studies on the combining properties of protamine and globin for insulin reported in part in a previous paper (33). These studies have been extended and will be considered in detail in a separate communication (42). The diurnal rhythm factor in diabetics (43) may also serve to aggravate the timing defects of globin insulin with zinc. There is a natural tendency for the blood sugar to fall during the day and rise during the night in a typical rhythmic fashion. The lowest levels are generally reached in the late afternoon or early evening and the highest levels by early morning. In other words, globin insulin with zinc, when administered in a single daily injection before breakfast, produces a strong effect when the blood sugar is at its lowest and, at most, a very weak effect when the blood sugar is at its highest.

\section{SUM MARY}

1. Each of five different insulin preparations (the "2:1 mixture," specially modified insulin types NPC-40 and NPH-50, standard protamine zinc insulin, and globin insulin with zinc) have been compared in each of 19 women patients with diabetes mellitus under routine but controlled conditions in the hospital. The blood and urinary sugar responses to each insulin have been determined in each patient over periods of several consecutive days (mode of five days).

2. The patients have been divided into two rough groups, the relatively stable group and the relatively unstable group, primarily on the basis of the variability of the blood and urinary sugar responses. In the stable group the variability was relatively low whereas in the unstable group it was excessively high. The insulins have been compared in each patient on their ability to control the extent of, or to influence the pattern of, this variability.

3. The insulins showed no differential ability to control the extent of the variability of blood and urinary sugar response or, in other words, stability. On the other hand, type of insulin did influence the pattern of this variability, but only in the unstable group. The three modifications of standard protamine zinc insulin (the 2:1 mixture, type NPC-40 and type NPH-50) produced a nearly level pattern in the variability in this group, i.e., no conspicuously high or low points. In contrast, globin insulin with zinc gave consistently high points in the morning and low points in the afternoon or evening while standard protamine zinc insulin produced consistently low points in the morning and high points in the afternoon or evening. The results indicate that clinical insulin comparisons without a consideration of stability may be very misleading.

\section{APPENDIX}

Let $w, x, y$ and $z$ denote the four blood sugar determinations for one patient on one day: then the intra-daily variation in blood sugar for that patient and day is defined to be:

$\log \left\{\left(w-\frac{t}{4}\right)^{2}+\left(x-\frac{t}{4}\right)^{2}+\left(y-\frac{t}{4}\right)^{2}+\left(z-\frac{t}{4}\right)^{2}\right\}$. where

$$
\mathrm{w}+\mathrm{x}+\mathrm{y}+\mathrm{z}=\mathrm{t} \text {. }
$$

Let $w^{\prime}, x^{\prime}, y^{\prime}$, and $z^{\prime}$ be corresponding urinary sugar 
determinations: then intra-daily variation in urinary sugar is defined to be:

$\log \left\{\left(w^{\prime}-\frac{t^{\prime}}{4}\right)^{2}+\left(x^{\prime}-\frac{t^{\prime}}{4}\right)^{2}+\left(y^{\prime}-\frac{t^{\prime}}{4}\right)^{2}\right.$

$$
\left.+\left(z^{\prime}-\frac{t^{\prime}}{4}\right)^{2}+1\right\}
$$

where

$$
\mathbf{w}^{\prime}+\mathrm{x}^{\prime}+\mathrm{y}^{\prime}+\mathrm{z}^{\prime}=\mathbf{t}^{\prime}
$$

Let $t_{1}, t_{2} \cdots t_{p}$ and $t_{1}{ }^{\prime}, t_{2}{ }^{\prime} \cdots t_{p}{ }^{\prime}$ be the daily totals in a "group" of $p$ days for blood and urinary sugar respectively. Then inter-daily variation in blood sugar is defined to be:

$$
\log \left\{\frac{1}{p-1}\left[\left(t_{1}-\bar{t}\right)^{2}+\left(t_{2}-\bar{t}\right)^{2}+\cdots+\left(t_{p}-\bar{t}\right)^{2}\right]\right\},
$$

where

$$
\overline{\mathrm{t}}=\frac{1}{\mathrm{p}}\left(\mathrm{t}_{1}+\mathrm{t}_{2}+\cdots+\mathrm{t}_{\mathrm{p}}\right) .
$$

Inter-daily variation in urinary sugar is defined to be:

$\log \left\{\frac{1}{(p-1)}\left[\left(\mathrm{t}_{1}{ }^{\prime}-\overline{\mathrm{t}}^{\prime}\right)^{2}+\left(\mathrm{t}_{2}{ }^{\prime}-\overline{\mathrm{t}}^{\prime}\right)^{2}+\cdots\right.\right.$

where

$$
\left.\left.+\left(t_{p}{ }^{\prime}-\bar{t}^{\prime}\right)^{2}\right]+1\right\} \text {. }
$$

$$
\bar{t}^{\prime}=\frac{1}{p}\left(t_{1}{ }^{\prime}+t_{2}{ }^{\prime}+\cdots+t_{p}{ }^{\prime}\right)
$$

\section{BIBLIOGRAPHY}

1. Joslin, E. P., Difficulties in the use of protamine zinc insulin. J. A. M. A., 1938, 110, 90.

2. Kepler, E. J., Clinical experiences with protamine zinc insulin. J. A. M. A., 1938, 110, 92.

3. Mark, M. F., Optimum time for administration of protamine zinc insulin. Arch. Int. Med., 1939, 64, 897.

4. Lawrence, R. D., and Archer, N., Some experiments with protamine insulinate. Brit. M. J., 1936, 1, 747.

5. Colwell, A. R., Izzo, J. L., and Stryker, W. A., Intermediate action of mixtures of soluble insulin and protamine zinc insulin. Arch. Int. Med., 1942, 69, 931.

6. Ulrich, H., Clinical experiments with mixtures of standard and protamine zinc insulins. Ann. Int. Med., 1941, 14, 1166.

7. Colwell, A. R., and Izzo, J. L., Protamine zinc insulin modified for accelerated action. J. A. M. A., 1943, 122, 1231.

8. Colwell, A. R., Effective insulin timing in diabetes. $M$. Clin. North America, 1947, 31, 327.

9. Colwell, A. R., Protamine insulin mixtures in the treatment of diabetes mellitus. New York State J. Med., 1947, 47, 1103.

10. Peck, F. B., Insulin mixtures and modifications. Proc. Am. Diabetes A., 1946, 6, 273.

11. Sprague, R. G., The use of various kinds of insulin. M. Clin. North America, 1946, 30, 933.

12. Peck, F. B., Symposium on diabetes; insulin mixtures and modifications. M. Clin. North America, 1947, 31,343 .
13. MacBryde, C. M., and Roberts, $H$. K., A new modified protamine zinc insulin: comparison with histone zinc insulin, clear, and standard protamine zinc insulin. J. Clin. Invest., 1943, 22, 791.

14. MacBryde, C. M., and Roberts, H. K., Modified protamine zinc insulin; an improvement on standard protamine zinc insulin; comparative studies on 62 diabetic patients. J. A. M. A., 1943, 122, 1225.

15. MacBryde, C. M., and Reiss, R. S., Modified protamine zinc insulin. Comparison with globin zinc insulin and insulin mixtures. J. Clin. Endocrinol., 1944, 4, 469.

16. Gabriele, A. J., and Marble, A., Clinical experience with a new modified protamine insulin (NPH-50). Am. J. Digest Dis., 1948, 16, 197.

17. Kirkpatrick, N. R., Experience with a new insulin. Proc. Staff Meet., Mayo Clinic, 1949, 24, 365.

18. White, P., Modified protamine insulin (NPH-50), a clinical report. J. A. M. A., 1949, 141, 312.

19. Reiner, L., Searle, D. S., and Lang, E. H., On hypoglycemic activity of globin insulin. J. Pharmacol. \& Exper. Therap., 1939, 67, 330.

20. Duncan, G. G., and Barnes, C. E., The action of globin insulin compared with that of crystalline; unmodified, and protamine zinc insulin. Am. J. M. Sc., 1941, 202, 553.

21. Bauman, L., Clinical experience with globin insulin. Am. J. M. Sc., 1939, 198, 475.

22. Bailey, C. C., and Marble, A., Histone zinc insulin, globin (zinc) insulin and clear protamine zinc insulin; comparative study of their action. J. A. M. A., 1942, 118, 683.

23. Mosenthal, H. O., Globin insulin with zinc in the treatment of diabetes mellitus. J. A. M. A., 1944, 125, 483.

24. Jackson, R. L., and McIntosh, C. B., Globin insulin with zinc in the treatment of children with diabetes mellitus. Proc. Am. Diabetes A., 1945, 5, 97.

25. Marks, H. E., A new globin insulin. The importance of carbohydrate distribution in the control of diabetes with the modified insulins. M. Clin. North America, 1940, 24, 649.

26. Sindoni, A., Jr., Fasting blood sugar vs. post-prandial blood sugar as observed in normal individuals, medical (non-diabetic) patients, and patients with diabetes. Am. J. Digest. Dis., 1946, 13, 178.

27. Roberts, J. T., and Yater, W. M., Comparison of the clinical use of protamine zinc insulin and globulin insulin in equal doses. Ann. Int. Med., 1947, 26, 41.

28. Lawrence, R. D., Globin-zinc-insulin; some experiments. Brit. M. J., 1943, 2, 103.

29. Malins, J. M., Globin insulin : clinical trial. Brit. M. J., 1945, 2, 318.

30. Protas, M., Comparative study of action of globin insulin with other forms of insulin. M. Ann. District of Columbia, 1944, 13, 254.

31. Rohr, J. H., and Colwell, A. R., Comparative time action of globin insulins. Arch. Int. Med., 1948, 82, 54. 
32. Rohr, J. H., and Colwell, A. R., Comparison of intermediate insulins. Proc. Am. Diabetes A., 1948, 8, 39.

33. Izzo, J. L., Studies on modified insulins. I. Comparison of time-action curves in stabilized patients with constant hyperglycemia and glycosuria. II. Comparison of solubility properties in water and sera. Proc. Am. Diabetes A., 1949, 9, 225.

34a. Somogyi, M., A new reagent for the determination of sugars. J. Biol. Chem., 1945, 160, 61.

b. Somogyi, M., Determination of blood sugar. Ibid, 69.

35. Shaffer, P. A., and Hartmann, A. F., Iodometric determination of copper and its use in sugar analysis. II. Methods for the determination of reducing sugars in blood, urine, milk, and other solutions. J. Biol. Chem., 1921, 45, 365.

36. Snedecor, G. W., Statistical Methods Applied to Experiments in Agriculture and Biology. The Iowa State College Press, Ames, Iowa, 1946, 4th edition.
37. Trimble, H. C., and Maddock, S. J., Fluctuations of the capillary blood sugar in normal young men during a 24 hour period (including a discussion of the effect of sleep and of mild exercise). J. Biol. Chem., 1929, 81, 595.

38. Himsworth, H. P., and Kerr, R. B., Insulin-sensitive and insulin-insensitive types of diabetes mellitus. Clin. Sci., 1939, 4, 119.

39. Falta, W., cited by Himsworth (38).

40. Woodyatt, R. T., Diabetes mellitus, in: Cecil, R. L., A Textbook of Medicine. W. B. Saunders Co., Philadelphia, 1937, 4th edition, p. 620.

41. Haunz, E. A., An approach to the problem of the "brittle" diabetic patient. J. A. M. A., 1950, 142, 168.

42. Izzo, J. L., and Kunz, W., To be published.

43. Izzo, J. L., Diurnal (24-hour) rhythm in diabetes mellitus. I. Diurnal variation in levels of glucose in blood and urine. Proc. Am. Diabetes A., 1949, 9, 245.

\section{SPECIAL NOTICE TO SUBSCRIBERS}

Post Offices will no longer forward the Journal when you move.

Please notify Journal of Clinical Investigation, Business Office, c/o Cincinnati General Hospital, Cincinnati 29, Ohio, at once when you have a change of address and do not omit the zone number if there is one. 